\title{
Chern Numbers, Quaternions, and Berry's Phases in Fermi Systems
}

\author{
J. E. Avron ${ }^{\star \star \star}$, L. Sadun ${ }^{\star \star \star}$, J. Segert ${ }^{\star \star}$ and B. Simon ${ }^{\star \star}$
}

Division of Physics, Mathematics and Astronomy, California Institute of Technology, Pasadena, CA 91125, USA

Abstract. Yes, but some parts are reasonably concrete.

\section{Table of Contents}

1. Introduction . . . . . . . . . . . . . . . . . . . . . 596

1.1 The Adiabatic Connection. . . . . . . . . . . . . . . 596

1.2 The Spectral Bundles of $H(\mathbf{B}) . \quad$. . . . . . . . . . . . . . . . . . 599

1.3 Quadrupole Interactions . . . . . . . . . . . . . . . 600

1.4 Topological Quantum Numbers. . . . . . . . . . . . . 600

2. Time Reversal and Quaternions . . . . . . . . . . . . . . 601

2.1 Quaternionic Vector Spaces . . . . . . . . . . . . . . 601

2.2 Quaternionic Structure Maps. . . . . . . . . . . . . . 603

2.3 Angular Momentum and Time Reversal . . . . . . . . . . 604

3. Quadrupole Hamiltonians . . . . . . . . . . . . . . . . 605

3.1 Rotational Symmetry . . . . . . . . . . . . . . . . 605

3.2 Simple Hamiltonians. . . . . . . . . . . . . . . . . 606

3.3 A Child's Garden of Matricial Miscelanea . . . . . . . . . 607

4. The Homotopy of Simple Matrices . . . . . . . . . . . . . 608

5. $J=3 / 2$ Quadrupole Hamiltonians . . . . . . . . . . . . . 612

5.1 SO(5) Symmetry . . . . . . . . . . . . . . . . . . 612

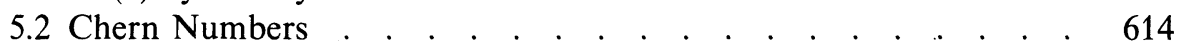

6. $J>3 / 2$ Quadrupole Hamiltonians . . . . . . . . . . . . . 615

6.1 The Chern-Simons Form . . . . . . . . . . . . . . . 615

6.2 Chern Numbers . . . . . . . . . . . . . . . . . . 616

6.3 Properties of the Adiabatic Connections . . . . . . . . . . 618

7. Holonomy of the Adiabatic Connection . . . . . . . . . . . 619

7.1 Symmetry Group Orbits . . . . . . . . . . . . . . . 620

$\star$ On leave form Physics Dept., Technion, Haifa, 32000, Israel

$\star \star$ Research partially supported by NSF Grant DMS- 8801918

$\star \star \star$ Address after September 1989: Courant Institute, New York University, NY, NY 10012, USA 
7.2 Split Spheres . . . . . . . . . . . . . . . . . . . 622

8. Appendix: $J=5 / 2$ Spectral Projections . . . . . . . . . . . . 625

\section{Introduction}

Quantum Hamiltonians that depend on parameters give rise to interesting geometric and topological questions [1-3]. A basic paradigm, due to M. Berry [1], is a spin $J$ in a magnetic field $\mathbf{B}$ :

$$
H(\mathbf{B})=\mathbf{B} \cdot \mathbf{J} .
$$

The geometric objects of concern are the bundles of one dimensional eigenspaces of $H(\mathbf{B})$ [2]. They are naturally defined over $\mathbf{B} \in \mathbf{R}^{3} /\{0\}$, with $\mathbf{B}=0$, the point of level crossings, removed. The adiabatic evolution can be used to define a natural connection for the bundles.

$H(\mathbf{B})$, being odd under time reversal, is the paradigm for the general case. It is natural to ask if time-reversal invariant Hamiltonians also give rise to interesting geometry. The answer is sensitive to statistics in the sense that bosons (i.e., integer spin systems) are different from fermions (i.e., half-odd-integer spin systems).

Mead [4] proposed the study of time-reversal invariant fermi systems, for which spin $J$ in a quadrupole electric field is the basic paradigm [5],

$$
H(Q)=Q_{\mu, v} J_{\mu} J_{v} .
$$

$Q_{\mu, v}$ are the components of a real, 3 by 3 , symmetric and traceless matrix, and we use the summation convention. In this paper, we compute the Chern numbers, curvatures, and holonomies for the bundles associated to spectral subspaces of $\{H(Q)\}$.

1.1 The Adiabatic Connection. Let $H(x)$ be

$$
H(x)=x_{\alpha} T_{\alpha},
$$

where $x=\left(x_{1}, \ldots, x_{n}\right) \in \mathbf{R}^{n} /\{0\}$ and the $\left\{T_{\alpha}\right\}$ are fixed self-adjoint operators.

Fix an eigenvalue $\lambda(x)$ of $H(x)$ and let $P(x)$ be the associated spectral projection:

$$
P(x)=\frac{1}{2 \pi i} \oint_{\Gamma} \frac{d z}{z-H(x)}
$$

where the contour $\Gamma$ circles $\lambda(x)$ counterclockwise in the complex $z$-plane. In the examples we consider here levels cross at $x=0$. On $\mathbf{R}^{n} /\{0\}, P(x)$ inherits the smoothness of $H(x)$, and in particular has fixed dimension. The adiabatic evolution transports vectors from the range of $P(x)$ to the range of $P\left(x^{\prime}\right)$. More precisely:

The adiabatic connection $A(P)$ is the operator-valued one-form ${ }^{1}$

$$
A(P)(x)=-[(d P)(x), P(x)] .
$$

\footnotetext{
${ }^{1}$ This definiton differs from the one of [6] by a factor of $i$
} 
Attention should be paid to the placement of parentheses. $(d P)$ is a operatorvalued form and does not differentiate succeeding expressions, in contrast to $d P=(d P)+P d$. To motivate the above definition, we state:

Theorem 1.1. (Kato [7]) Let $c$ be a path of Hamiltonians parametrized by t. Let $U_{A}(t)$ be the solution of

$$
\left(\frac{\partial}{\partial t}-\left[\frac{\partial P}{\partial t}, P\right]\right) U_{A}(t)=0
$$

where $P(t)=P(c(t))$, with initial condition $U_{A}(0)=1$. Then $U_{A}(t)$ is a unitary operator which maps the range of $P(0)$ to the range of $P(t)$, i.e.,

$$
P(t) U_{A}(t)=U_{A}(t) P(0) \text {. }
$$

Remarks.

1. It is a result of Kato that the physical evolution, generated by $H(x)$, reduces to the adiabatic evolution (1.6), in the adiabatic limit $[7,6]$.

2. For closed paths, with $c(1)=c(0), U_{A}(1)$ is a unitary map from the range of $P(0)$ to itself, and may be identified with an element of $U(n)$, where $n=\operatorname{dim}(P)$. This is the adiabatic holonomy. When $\operatorname{dim}(P)=1$, the holonomy is Berry's phase.

The adiabatic curvature two-form $\Omega(P)$ is defined as the curvature of the adiabatic connection in the usual way, as the adiabatic holonomy over small closed paths $[8,9]$.

\section{Proposition 1.2.}

a) On Hilbert space-valued differential forms satisfying $P(x) B(x)=B(x)$, the covariant derivative corresponding to the adiabatic connection is

$$
\nabla \equiv d+A=P d
$$

b) The adiabatic curvature is

$$
\Omega(x)=P(d P)(d P) P .
$$

c) In a neighborhood of $P_{0}$, given a smooth family of unitaries $U(x)$ such that $P(x)=U(x) P_{0} U(x)^{-1}$, the curvature is given by

$$
\Omega=U\left(d \tilde{A}+\tilde{A}^{2}\right) U^{-1}, \quad \tilde{A} \equiv P_{0} U^{-1}(d U) P_{0} .
$$

Proof. a) follows from [2] and [10]. Part b) is a computation based on the equality $\Omega \equiv \nabla^{2}$. Part c) is direct computation.

Remarks.

1. The proposition can be understood in terms of gauge transformations. Consider $\widetilde{B}(x)=U^{-1}(x) B(x)$. On Hilbert space-valued forms $\widetilde{B}(x)$ satisfying $P_{0} \widetilde{B}(x)=\widetilde{B}(x)$, the covariant derivative transforms to $\tilde{\nabla}=U(x)^{-1} \nabla U(x)=d+\tilde{A}$.

2. Equation (1.6) is equivalent to $\nabla_{\partial / \partial t} U_{A}=0$.

Time reversal is associated with an antiunitary operator $\Theta$. We shall return to this in Sect. 2. For the curvature, using (1.4) and (1.9), this has the following consequence: 
Proposition 1.3. Suppose $H(x) \Theta=\Theta H(x)$, then $\operatorname{Tr}\left(\Omega^{2 n+1}\right)=0$. In particular, if $\operatorname{dim}(P)=1$, then the adiabatic curvature vanishes identically.

Proof. Since $\Theta H=H \Theta$, then also $\Theta P=P \Theta$, and $\Theta \Omega \Theta^{-1}=\Omega$. Since for any complex-linear operator $M$ we have $\operatorname{Tr}\left(\Theta M \Theta^{-1}\right)=\operatorname{Tr}\left(M^{\dagger}\right)$, it follows that

$$
\operatorname{Tr}\left(\Omega^{2 n+1}\right)=\operatorname{Tr}\left(\Theta \Omega^{2 n+1} \Theta^{-1}\right)=\operatorname{Tr}\left(\left(\Omega^{2 n+1}\right)^{\dagger}\right)=-\operatorname{Tr}\left(\Omega^{2 n+1}\right)=0 .
$$

For bosons, $\operatorname{dim}(P)=1$ generically, so time reversal invariant bosons are uninteresting. Fermions, in contrast, have Kramers degeneracy, which allows for nonzero $\Omega$.

We now present some prerequisites for the introduction of Chern classes.

\section{Proposition 1.4.}

a)

b)

$$
d \operatorname{Tr}\left[\Omega^{m}\right]=0
$$

$$
\boldsymbol{\Omega}^{\dagger}=-\boldsymbol{\Omega}
$$

Proof. a) follows from the fact that $(d P)$ maps the range of $P$ to the range of $(1-P)$, and vice-versa, so any form of odd degree made of $P$ and $(d P)$ has zero trace. $b)$ is a consequence of Eq. (1.9) the anticommutativity of one-forms.

Topological invariants arise from the fact that parameter space has nontrivial topology once points of level crossing are removed. In particular, for the examples we consider parameter space is $\mathbf{R}^{n} /\{0\},(n=3$ and $n=5$ for $H(\mathbf{B})$ and $H(Q)$ respectively), which is noncontractible. Bundles over parameter space may then have nontrivial Chern classes, with corresponding nonzero Chern integers. More precisely:

Definition 1.5. ([11,8])

a) The $m$-th Chern class is

$$
\omega_{m}=\frac{(-1)^{m}}{(2 \pi i)^{m} m !} \operatorname{Tr}\left(\Omega^{m}\right)
$$

b) The $m$-th Chern number, $C_{m}(P, X)$ associated with the projection $P$ and the closed $2 m$-dimensional submanifold $X$ in parameter space, is

$$
C_{m}(P, X)=\int_{X} \omega_{m}(P)
$$

For the examples we consider, where parameter space is $\mathbf{R}^{n} /\{0\}$ the only relevant closed surfaces are $S^{n-1}$, i.e., the 2-sphere for $H(\mathbf{B})$ and the 4-sphere for $H(Q)$.

Proposition 1.6. Consider a family of Hamiltonians $H(x)$ of the form (1.3), with $n=2 m+1$, and suppose further that $H(x)$ is nondegenerate for all $x \neq 0$. Denote the projection onto the $\mu$-th eigenvalue of $H(x)$ by $P_{\mu}(x)$, with $\mu=1, \ldots, v$. Then

$$
C_{m}\left(P_{\mu}, S^{2 m}\right)=-C_{m}\left(P_{v+1-\mu}, S^{2 m}\right) .
$$

Proof. The map $H(x) \rightarrow H(-x)$ reverses the order of the eigenvalues, and so 
interchanges $P_{\mu}$ and $P_{v+1-\mu}$. The antipodal map $x \rightarrow-x$ on $S^{2 m} \subset \mathbf{R}^{2 m+1}$ is orientation-reversing, and changes the sign of $\Omega^{m}$.

1.2 The Spectral Bundles of $H(\mathbf{B})$. We now derive the well known properties of the spectral bundles of $\{H(B)\}$. We use a method that will be generalized to $\{H(Q)\}$. The holonomy formulae will in addition be used in calculating the holonomy of $\{H(Q)\}$. The spectrum of $H(\mathbf{B})$ is

$$
|\mathbf{B}|\{-J,-J+1, \ldots, J\} .
$$

$H(\mathbf{B})$ is nondegenerate away from $\mathbf{B}=0$. Denote the spectral projections by $P_{m}(\mathbf{B})$, where $m$ is the eigenvalue of $H(\hat{\mathbf{B}})$, with $\hat{\mathbf{B}}=\mathbf{B} /|\mathbf{B}|$.

A special feature of $H(\mathbf{B})$ is that the results can be inferred from the action of $S O(3)$ rotations. For example, $P_{m}(\hat{\mathbf{B}})$ is unitarily equivalent to $P_{m}(\hat{\mathbf{n}})$, with $\hat{\mathbf{n}}$ denoting the north pole, by any unitary operator corresponding to the rigid rotation of $\hat{\mathbf{n}}$ to $\hat{\mathbf{B}}$.

Proposition 1.7. $([1,2])$

a) $\omega_{1}$ for each eigenvalue is rotationally invariant.

b)

$$
C_{1}\left(P_{m}, S^{2}\right)=-2 m
$$

c) For a closed loop $c(t)$ on $S^{2}, c(1)=c(0)$,

$$
U_{A}(1) P_{m}=\exp (-i m S) P_{m},
$$

where $S$ is the solid angle enclosed by $c$.

The remainder of this section is a proof of this proposition. We use only the symmetry by rotations about the $z$-axis, not the full rotation symmetry actually present in $H(\mathbf{B})$.

Using formula (1.10) and the cyclicity of the trace, we find locally

$$
\operatorname{Tr}(\Omega)=\operatorname{Tr}\left(d \tilde{A}+\tilde{A}^{2}\right)=\operatorname{Tr}(d \tilde{A})=d \operatorname{Tr}(\tilde{A}) .
$$

Let $(\theta, \phi)$ be the usual spherical coordinates. Let $P_{0}=P_{(0,0)}=|m\rangle\langle m|$. Choose for each $0<\theta<\pi$ a unitary operator $V_{\theta}$ such that $P_{(\theta, 0)}=V_{\theta} P_{0} V_{\theta}^{-1}$. Define $W_{\phi}=\exp \left(-i \phi J_{3}\right)$. Then

$$
P_{(\theta, \phi)}=W_{\phi} V_{\theta} P_{0} V_{\theta}^{-1} W_{\phi}^{-1} .
$$

Define $U=W V . \tilde{A}$ is globally well defined away from the poles, ${ }^{2}$ and

$$
\operatorname{Tr}(\Omega)=d \operatorname{Tr}(\tilde{A})=d \operatorname{Tr}\left(P_{\theta} W^{-1}(d W)\right)=-i d\left[\operatorname{Tr}\left(P_{\theta} J_{3}\right) d \phi\right] .
$$

Let $N \subset S^{2}$ be the set consisting of the north and south poles. The first Chern number is then

\footnotetext{
${ }^{2} U(\phi+2 \pi)=\exp \left(-2 \pi i J_{3}\right) U(\phi)$. However, even if $\exp \left(-2 \pi i J_{3}\right)=-1$, we have $\tilde{A}(\theta, \phi+2 \pi)=\tilde{A}(\theta, \phi)$
} 


$$
\begin{aligned}
C_{1} & =\frac{1}{2 \pi} \int_{S^{2} / N} d\left[\operatorname{Tr}\left(P_{\theta} J_{3}\right) d \phi\right]=\lim _{\varepsilon \rightarrow 0}\left(\operatorname{Tr}\left(P_{(\pi-\varepsilon)} J_{3}\right)-\operatorname{Tr}\left(P_{\varepsilon} J_{3}\right)\right) \\
& =\operatorname{Tr}\left(P_{\pi} J_{3}\right)-\operatorname{Tr}\left(P_{0} J_{3}\right) .
\end{aligned}
$$

For Berry's example, $P_{0}=|m\rangle\left\langle m\left|, P_{\pi}=\right|-m\right\rangle\langle-m|$, and $C_{1}=-2 m$. (Some authors use a different sign convention in the definition of first Chern class, (1.14).)

For a connection with an Abelian holonomy group, $U_{A}(1)$ in this example, the adiabatic transport $U_{A}(1)$ for a closed path $c, c(1)=c(0)$, which does not go through either pole, and for which $U(c(t))=W V(c(t))$ is single-valued ${ }^{3}$, equals

$$
U_{A}(1)=\exp \left(\int_{c} \operatorname{Tr}(\tilde{A})\right)=\exp \left(-i \int_{c}\left(P_{\theta} J_{3}\right) d \phi\right) .
$$

For a connection with Abelian holonomy group, $U_{A}(1)$ can also be expressed as the exponential of the integral of the curvature over a disk bounded by the path c. By Stokes' theorem, this is the same as Eq. (1.24).

For Berry's example, $H(\mathbf{B})$, using the remaining rotational symmetry, we take $V_{\theta}=\exp \left(-i \theta J_{2}\right)$

$$
\operatorname{Tr}\left(P_{\theta} J_{3}\right)=\operatorname{Tr}\left(P_{0} \exp \left(i \theta J_{2}\right) J_{3} \exp \left(-i \theta J_{2}\right)\right)=\cos (\theta) \operatorname{Tr}\left(P_{0} J_{3}\right)
$$

Here we used the fact $\operatorname{Tr}\left(P_{0} J_{1}\right)=\left\langle m\left|J_{1}\right| m\right\rangle=0$. The adiabatic time evolution around the closed loop gives a phase equal to $-i \operatorname{Tr}\left(P_{0} J_{3}\right)$ multiplied by the solid angle bounded by the loop $[1,2]$.

1.3 Quadrupole Interactions. Spins do not couple directly to electric fields. Up to an overall constant (reduced matrix element) for each $J$-multiplet, the form of the interaction Hamiltonian is apparent from the Wigner-Eckart theorem [13]. We outline why the interaction Hamiltonian does not vanish.

Consider perturbing an attom by a quadrupole electric field with sources at infinity. Such a field is described by a 3 by 3 symmetric and traceless matrix $Q$. $Q \mathbf{x}$ is the electric field at $\mathbf{x} \in \mathbf{R}^{3}$. For each electron the perturbation is [14]

$$
\frac{1}{2} Q_{\mu, v} x_{\mu} x_{v}+\frac{e}{4 m^{2}} \vec{\sigma} \cdot(Q \mathbf{x}) \times(-i \vec{\nabla})
$$

The first term is the usual potential energy term and the second has the same origin as the spin-orbit interaction term.

1.4 Topological Quantum Numbers. Quantum numbers that come from symmetry of an operator play two roles: They label the spectrum, and give selection rules for transitions. Selection rules forbid certain transitions. The Chern numbers play analogous roles for families of operators. They clearly label the spectrum. They

\footnotetext{
${ }^{3}$ For half-odd-integral, $J, U$ is double-valued on $S^{2}-N$, although $\tilde{A}$ is single-valued. The following equation is then only valid for closed paths which wrap around an even number of times. Alternatively, the substitution $J_{3} \rightarrow J_{3}+\frac{1}{2}$ makes $U$ single-valued, and makes the following equation valid in full generality. See Zee [12] for further discussion of this point
} 
also give selection rules for adiabatic processes in the sense that a nonzero Chern number guarantees nontrivial holonomy for some paths. In the nondegenerate case the holonomy is just a phase. For time-reversal invariant fermions the holonomy is an $S U$ (2) matrix, so the initial and final state can be orthogonal. For example, as we shall see, appropriate closed paths in the space of quadrupoles can cause adiabatic evolution of a $J_{3}=3 / 2$ eigenstate to a $J_{3}=-3 / 2$ eigenstate.

\section{Time Reversal and Quaternions}

It is an old result of Wigner [15] that time reversal in quantum mechanics is implemented by an antiunitary operator $\Theta$ such that $\Theta^{2}=1$ for bosons and $\Theta^{2}=-1$ for fermions. Such a $\Theta$ can be written as $\Theta=U \circ *$ with $*$ denoting complex conjugation and with $U$ unitary. In group representation theory, $\Theta$ is known as a structure map, and traces its origins to the work of Frobenius and Schur [16].

In this section we collect some standard facts about time reversal for fermions. For original works, see $[17,15,18]$. That time-reversal is identified with an antiunitary can be seen from the basic requirements that the coordinate $\mathbf{x}$ and the momentum $p$ be even and odd, respectively, under time reversal. From the canonical commutation relation $[\mathbf{p} \cdot \mathbf{a}, \mathbf{x} \cdot \mathbf{b}]=-i \mathbf{a} \cdot \mathbf{b}$, with $\mathbf{a}, \mathbf{b} \in \mathbf{R}^{n}$, it follows that time-reversal anticommutes with $i$, and so is antilinear.

The distinction between integer and half-odd-integer spins comes from the oddness of angular momentum $\mathbf{J}$ under time reversal; $\Theta \mathbf{J}=-\mathbf{J} \Theta$. Note that $H(Q) \Theta=\Theta H(Q)$ trivially follows. In the usual representation, where $J_{1}$ and $J_{3}$ are real, while $J_{2}$ is imaginary, the unitary in $\Theta$ is a rotation by $\pi$ about the $y$-axis:

$$
\Theta=\exp \left(-i \pi J_{2}\right) \circ * \text {. }
$$

For an irreducible representation, this choice is unique up to an overall phase. $\exp \left(-i \pi J_{2}\right)$ is real, so it commutes with $* . \Theta^{2}=\exp \left(-2 \pi i J_{2}\right)$ is a $2 \pi$ rotation, so $\Theta^{2}=1$ for bosons and -1 for fermions. Note that $\Theta^{2}$ does not depend on the choice of overall phase, because $\Theta$ is antilinear.

Antiunitary operators whose squares are -1 , acting on a finite-dimensional Hilbert space, exist only when the space is even-dimensional. For every vector $|v\rangle$, $\Theta|v\rangle$ is orthogonal to $|v\rangle$ since

$$
\langle v \mid \Theta v\rangle=\left\langle\Theta^{2} v \mid \Theta v\right\rangle=-\langle v \mid \Theta v\rangle=0,
$$

where we have used the fact that $\langle u \mid v\rangle=\langle\Theta v \mid \Theta u\rangle$.

An immediate consequence of this is Kramer's degeneracy: if $H$ is a hermitian matrix commuting with $\Theta$, then each eigenvalue has degeneracy of even degree [19].

2.1 Quaternionic Vector Spaces. Dyson [17] pointed out that fermionic timereversal can be interpreted as a quaternionic structure. The basic ideas of this observation date back to Frobenius and Schur [16]. We recall first the basic facts about quaternions, and then recall the quaternionic interpretation of time-reversal.

Definition 2.1.

a) The noncommutative field (or division algebra) $\mathbf{H}$ of quaternions is generated 
as a real algebra over $\mathbf{R}$ by the elements $i, j, k$, with

$$
i^{2}=j^{2}=k^{2}=i j k=-1 \text {. }
$$

b) The conjugate of $x=x_{0}+x_{1} i+x_{2} j+x_{3} k \in \mathbf{H}$, denoted $\bar{x}$, is

$$
\bar{x}=x_{0}-x_{1} i-x_{2} j-x_{3} k \text {. }
$$

c) The norm $|\cdot|$ on $\mathbf{H}$ is defined by

$$
|x|^{2}=x \bar{x}
$$

The unit quaternions are thus the 3 -sphere $S^{3}$. Note also that $i \rightarrow i \sigma_{3}, j \rightarrow-i \sigma_{2}$, $k \rightarrow-i \sigma_{1}$, with $\sigma_{1}, \sigma_{2}, \sigma_{3}$ the Pauli matrices, are a representation of the quaternion algebra.

When considering quaternionic vector spaces, the noncommutativity of the quaternions forces us to mind the distinction between right and left multiplication by scalars.

Definition 2.2. A quaternionic vector space $V$ is defined by the following properties: $v, w \in V, x, y \in \mathbf{H}$

$$
(v+w) x=v x+w x, \quad v(x+y)=v x+v y, \quad v(x y)=(v x) y .
$$

We have chosen to have scalar multiplication of a vector by a quaternion act on the right. This choice of right multiplication by scalars yields a simple formula for multiplication of quaternion-valued matrices, as we shall see. Left multiplication of vectors by scalars is not defined, and expressions like " $x v$ " have no basisindependent meaning.

A quaternionic operator $H$ on $V$ is a quaternionic linear map of $V$ into itself:

$$
H[(v+w) x]=(H v) x+(H w) x .
$$

For a choice of basis in $V$, a quaternionic operator has a matrix expression,

$$
(H v)_{\alpha}=H_{\alpha, \beta} v_{\beta},
$$

where the matrix elements $H_{\alpha, \beta} \in \mathbf{H}$ and summation over repeated indices is implied. Let $A$ and $B$ be two quaternionic operators on $V$. Then

$$
(A B v)_{\alpha}=A_{\alpha, \beta} B_{\beta, \gamma} v_{\gamma}, \quad(A B)_{\alpha, \gamma}=A_{\alpha, \beta} B_{\beta, \gamma} .
$$

If we had instead chosen left scalar multiplication in (2.6), this would have had the unfortunate consequence that $(A B)_{\alpha, \gamma}$ would equal $B_{\beta, \gamma} A_{\alpha, \beta}$.

We now consider the quaternionic inner product on $V$. This is a sesquilinear map $V \times V \rightarrow H$ with the properties

$$
(v x, w y)=\bar{x}(v, w) y, \quad(v, w)=\overline{(w, v)} .
$$

Definition 2.3. The quaternionic adjoint $H^{*}$ of an operator $H$ on a quaternionic vector space with inner product is defined by

$$
\left(H^{*} w, v\right)=(w, H v)
$$

for all $v, w \in V$. If we use a basis $\left\{e_{l}\right\}$ to identify $V$ with $\mathbf{H}^{n}$, and take the canonical 
inner product on $\mathbf{H}^{n}$ defined by

$$
\left(e_{l}, e_{m}\right)=\delta_{l, m},
$$

then the matrix representative of the adjoint operation is

$$
\left(H^{*}\right)_{\alpha, \beta}=\overline{\left(H_{\beta, \alpha}\right)} \text {. }
$$

2.2 Quaternionic Structure Maps. A convenient formalism of dealing with quaternionic vector spaces is that of structure maps on complex vector space $[20,21]$. A structure map $\Theta$ on a complex vector space $W$ is an antilinear map satisfying $\Theta^{2}= \pm 1$.

We consider the case $\Theta^{2}=-1$, which we call a quaternionic structure map. A right quaternionic action on $W$ is defined by

$$
v i \equiv i v, \quad v j \equiv \Theta v, \quad v k \equiv(v i) j=\Theta(i v)=-i \Theta v, \quad v \in W .
$$

The first equation simply means that right multiplication of a vector by the quaternion $i$ is the same as multiplication by the complex number $i$. A quaternioniclinear operator $A$ must commute with the right action of $i$, which makes it a complex-linear operator, and with the right action of $j$, which means $A \Theta=\Theta A$. It then automatically commutes with the right action of $k$.

When $W$ is given a quaternionic basis, quaternionic linear operators can be written as matrices with quaternion entries. A quaternionic basis of $W$ is a set of vectors $e_{l} \in W$ such that $\left\{e_{l}, \Theta e_{l}\right\}$ is a basis of $W$ as a complex vector space.

Decomposing a vector $v$ into quaternionic components $v_{l}$ (such that $v=e_{l} v_{l}$ ), we further decompose each quaternionic component, $v_{l}=v_{l, 0}+v_{l, 1} i+v_{l, 2} j+v_{l, 3} k$ with $v_{l, \alpha}$ real, and note that

$$
v=e_{l}\left(v_{l, 0}+v_{l, 1} i+v_{l, 2} j+v_{l, 3} k\right)=e_{l}\left(v_{l, 0}+v_{l, 1} i\right)+\left(\Theta e_{l}\right)\left(v_{l, 2}-v_{l, 3} i\right)
$$

A quaternionic operator is determined by its action on the complex basis, $\left\{e_{l}, \Theta e_{l}\right\}$. As an example, we look at a one-quaternionic-dimensional vector space. [i] denotes the 1 by 1 matrix with entry $i, A_{i}$ denotes the corresponding 2 by 2 complex matrix in the basis $(e, \Theta e)$,

$$
\begin{gathered}
{[i] 1=i, \quad \Rightarrow \quad A_{i} e=i e} \\
{[i] j=i j=k, \quad \Rightarrow \quad A_{i}(\Theta e)=-i \Theta e,}
\end{gathered}
$$

and so

$$
A_{i}=\left(\begin{array}{rr}
i & 0 \\
0 & -i
\end{array}\right)=i \sigma_{3}
$$

Similarly, we compute

$$
A_{j}=\left(\begin{array}{rr}
0 & -1 \\
1 & 0
\end{array}\right)=-i \sigma_{2} \quad A_{k}=\left(\begin{array}{rr}
0 & -i \\
-i & 0
\end{array}\right)=-i \sigma_{1} .
$$

The matrix corresponding to a time-reversal invariant operator $B$ relative to an orthonormal basis $\left\{e_{l}, \Theta e_{l}\right\}$ consists of $2 \times 2$ blocks which are real linear 
combinations of $\left\{1, A_{i}, A_{j}, A_{k}\right\}$. This may be thought of as a quaternion-valued matrix.

If we have a sesquilinear complex-valued inner product on $V$, and if $\Theta$ is antiunitary with respect to this inner product, then we can express the quaterionvalued inner product as ${ }^{4}$

$$
(v, w)=\langle v \mid w\rangle-\langle v \mid \Theta w\rangle j .
$$

2.3 Angular momentum and Time Reversal. Let $|J, m\rangle, m=-J, \ldots, J$ be the standard basis for a spin $J$ multiplet, i.e.,

$$
\begin{aligned}
\mathbf{J}^{2}|J, m\rangle & =J(J+1)|J, m\rangle, \\
J_{3}|J, m\rangle & =m|J, m\rangle, \\
J_{ \pm}|J, m\rangle & =\left(J_{1} \pm i J_{2}\right)|J, m\rangle=\sqrt{J(J+1)-m(m \pm 1)}|J, m \pm 1\rangle .
\end{aligned}
$$

Time-reversal is implemented as in (2.1), and we have

\section{Proposition 2.4.}

$$
\Theta \alpha|J, m\rangle=(-)^{(J-m)} \alpha^{*}|J,-m\rangle, \quad \alpha \in \mathbf{C} .
$$

Proof. $[15,13]$ We evaluate $\exp \left(-i \pi J_{2}\right)|J, m\rangle$. Since $\Theta J_{3} \Theta^{-1}=-J_{3}$, it follows that $\Theta|J, m\rangle=\beta_{m}|J,-m\rangle$ for some phase $\beta_{m}$. Since $J_{2}$ is purely imaginary, $\exp \left(-i \pi J_{2}\right)$ is a real matrix, and $\beta_{m}= \pm 1$. Now $\Theta J_{+}=-J_{-} \Theta$, so $\beta_{m}=-\beta_{m \pm 1}$. It then suffices to determine $\beta_{J}$.

We build up the spin $J$ representation from tensor products of $2 J$ distinct spin $\frac{1}{2}$ representations. We label the generators of the $n$-spin $\frac{1}{2}$ representation by $\left(J_{1, n}, J_{2, n}, J_{3, n}\right)$, and the usual (real) eigenstates of $J_{3, n}$ by $\left|\frac{1}{2}, \pm m\right\rangle_{n}$. Then recall that

$$
|J, \pm J\rangle=\left|\frac{1}{2}, \pm \frac{1}{2}\right\rangle_{1} \otimes\left|\frac{1}{2}, \pm \frac{1}{2}\right\rangle_{2} \otimes \cdots\left|\frac{1}{2}, \pm \frac{1}{2}\right\rangle_{2 J} .
$$

Now for $\operatorname{spin} \frac{1}{2}$ we have $\exp \left(-i \pi J_{2}\right)=-i \sigma_{2}$, and so

$$
\exp \left(-i \pi J_{2, l}\right)\left|\frac{1}{2}, \frac{1}{2}\right\rangle_{l}=+\left|\frac{1}{2},-\frac{1}{2}\right\rangle_{l} \text {. }
$$

We conclude that

$$
\exp \left(-i \pi J_{2}\right)|J, J\rangle=|J,-J\rangle
$$

and so $\beta_{J}=+1$ and $\beta_{m}=(-1)^{J-m}$.

The preceding proposition suggests the nonstandard complex basis,

$$
\begin{aligned}
|\alpha\rangle & =\alpha_{1}|J, J\rangle+\alpha_{2} \Theta|J, J\rangle+\alpha_{3}|J, J-2\rangle+\alpha_{4} \Theta|J, J-2\rangle \cdots \\
& =\alpha_{1}|J, J\rangle+\alpha_{2}|J,-J\rangle+\alpha_{3}|J, J-2\rangle+\alpha_{4}|J, 2-J\rangle \cdots
\end{aligned}
$$

and an associated quaternionic basis:

\footnotetext{
${ }^{4}$ If $A$ is a complex-linear hermitian operator invariant under time reversal, then $\operatorname{Tr}_{\mathrm{c}}(A)=2 \operatorname{Tr}_{H}(A) \in \mathbf{R}$. For non-hermitian operators, $\operatorname{Tr} A \notin \mathbf{R}$ in general. $\Omega^{2 n}$ is Hermitian, so the $2 n$-th Chern classes can be defined from purely quaternionic information, and are often called symplectic Pontrjagin classes in this context
} 
Definition 2.5. The canonical quaternionic basis of the half-odd-integer $J$ Hilbert space is

$$
e_{l} \equiv|J, J-2 l+2\rangle, \quad l=1, \ldots, J+\frac{1}{2}
$$

\section{Quadrupole Hamiltonians}

3.1 Rotational Symmetry. We first present the action on the rotation group on the space of quadrupoles, following the outline of [22]. A quadrupole $Q$ is a $3 \times 3$ real symmetric matrix with zero trace. The space of quadrupoles is a five-dimensional real vector space, with an inner product $\left(Q, Q^{\prime}\right)=\frac{3}{2} \operatorname{Tr}\left(Q Q^{\prime}\right)$. A unit quadrupole satisfies $\frac{3}{2} \operatorname{Tr} Q^{2}=1$. A convenient orthonormal basis is given by ${ }^{5}$

$$
\begin{aligned}
& Q_{0}=\frac{1}{3}\left(\begin{array}{lll}
-1 & & \\
& -1 & \\
& & 2
\end{array}\right) \text {, } \\
& Q_{1}=\frac{1}{\sqrt{3}}\left(\begin{array}{lll} 
& & 1 \\
& 0 &
\end{array}\right), \quad Q_{2}=\frac{1}{\sqrt{3}}\left(\begin{array}{lll}
0 & & \\
& 0 & 1 \\
& 1 & 0
\end{array}\right), \\
& Q_{3}=\frac{1}{\sqrt{3}}\left(\begin{array}{lll}
1 & & \\
& -1 & \\
& & 0
\end{array}\right), \quad Q_{4}=\frac{1}{\sqrt{3}}\left(\begin{array}{lll}
0 & 1 & \\
1 & 0 & \\
& & 0
\end{array}\right) \text {. }
\end{aligned}
$$

On the sphere of unit quadrupoles, we shall call $+Q_{0}$ the north pole, and $-Q_{0}$ the south pole.

The rotation group $S O(3)$ acts on the space of quadrupoles by $Q \rightarrow R Q R^{-1}$, preserving the inner product. This is of course the irreducible real $J=2$ representation. The space of diagonal quadrupole matrices is two dimensional, spanned by $Q_{0}$ and $Q_{3}$. Every unit quadrupole is rotationally related to a diagonal unit quadrupole, i.e., a matrix of the form $Q_{\theta}=\cos (\theta) Q_{0}+\sin (\theta) Q_{3}$,

$$
Q_{\theta}=\frac{2}{3}\left(\begin{array}{lll}
\cos (\theta+2 \pi / 3) & & \\
& \cos (\theta-2 \pi / 3) & \\
& & \cos (\theta)
\end{array}\right),
$$

for some value of $0 \leqq \theta<2 \pi$. In fact, we can restrict $\theta$ to a subset of the circle:

\section{Proposition 3.1.}

a) Every unit quadrupole is rotationally related to exactly one $Q_{\theta}$ with $0 \leqq \theta \leqq \pi / 3$. In particular, the south pole $-Q_{0}$ is rotationally related to $Q_{\pi / 3}$.

b) The orbits of $Q_{\theta}$ are two-dimensional when $\theta=n \pi / 3$ for any integer $n$, and three-dimensional otherwise.

c) For $0 \neq n \pi / 3$, the subgroup of $S O(3)$ which leaves $Q_{\theta}$ invariant is the dihedral

${ }^{5}$ We retain the normalization of [5], which was chosen because it is particularly convenient for $J=3 / 2$ 
group $D_{2}$. The lift $D_{2}$ to the $S U(2)$ double cover is the eight-element group generated by the unit quaternions $\{i, j, k\}$.

Proof. it a): We denote by $L_{1}, L_{2}$, and $L_{3}$ the usual complex Hermitian generators of $S O(3)$. Then $-i L_{1},-i L_{2}$ and $-i L_{3}$ are real and antisymmetric. The rotation $\exp \left( \pm(2 \pi / 3)(i / \sqrt{3})\left(L_{1}+L_{2}+L_{3}\right)\right)$ cyclically permutes the diagonal entries of $Q_{\theta}$, so $Q_{\theta}$ is rotationally related to $Q_{(\theta \pm 2 \pi / 3)}$. Now the rotation $\exp \left(\pi(i / 2) L_{3}\right)$ permutes the first two diagonal entries of $Q_{\theta}$, so $Q_{\theta}$ is rotationally related to $Q_{-\theta}$. Thus any unit quadrupole is rotationally related to some $Q_{\theta}$, with $0 \leqq \theta \leqq \pi / 3$. The $\theta$ in this interval is unique, because $\operatorname{Det}\left(Q_{\theta}\right)=\frac{2}{27} \cos (3 \theta)$ is a one-to-one function on this interval.

Part b) is checked by noting that $Q_{0}$ and $Q_{\pi / 3}$ each commute with exactly one generator of the rotation group, while $Q_{\theta}$ for $0<\theta<\pi / 3$ does not commute with any nonzero linear combination of the generators.

Part c) is standard, see $[15,13]$.

3.2 Simple Hamiltonians. We say that a hermitian linear operator $M$ over a field $\mathbf{F}=\mathbf{R}, \mathbf{C}, \mathbf{H}$ is simple if it has no degenerate eigenvalues. For fermi systems, time-reversal-invariant operators are never simple over $\mathbf{C}$ because of Kramer's degeneracy. They may, however, be simple when thought of as quaternionic operators.

We now show that $H(Q)$ is quaternionically simple for $Q \neq 0$, assuming of course $J$ is half-odd integral. If this condition were not satisfied, the spectral bundles, and consequently the Chern numbers would not be well-defined. We shall use the notation

$$
T_{\alpha}=H\left(Q_{\alpha}\right), \quad \alpha=0, \ldots, 4,
$$

so $T_{0}=J_{3}^{2}-\mathbf{J}^{2} / 3$, etc. We also define

$$
T_{\theta}=\cos (\theta) T_{0}+\sin (\theta) T_{3} .
$$

The basic result we will use is:

Proposition 3.2. Let $M$ be a hermitian $n \times n$ matrix with values in $\mathbf{F}=\mathbf{R}, \mathbf{C}, \mathbf{H}$. Suppose further $M$ is tridiagonal, with no zeros on the subdiagonal and superdiagonal. Then $M$ is F-simple.

Proof. Suppose $M$ has a degenerate eigenvalue $\lambda$. Then the eigenspace of $\lambda$ contains a nonzero vector $v$ with $v_{1}=0$. Since $M$ is tridiagonal,

implying $v_{2}=0$. Then

$$
0=\lambda v_{1}=(H v)_{1}=H_{12} v_{2},
$$

$$
0=\lambda v_{2}=(H v)_{2}=H_{23} v_{3} \text {, }
$$

implying $v_{3}=0$, and so on. Thus we get $v=0$, a contradiction, and so $M$ must be simple.

Let $W(R)$ be the representative of the rotation $R$. This is defined only up to 
an overall sign, since the representation has half-odd-integral $J$. Then

$$
W(R) H(Q) W(R)^{-1}=H\left(R Q R^{-1}\right),
$$

with no sign ambiguity. Combining (3.7) with Proposition 3.1, we obtain:

Proposition 3.3. For $Q$ a unit quadrupole, $H(Q)$ there exists a unitary $W(R)$ corresponding to a rotation $R$ such that

$$
W(R) H(Q) W(R)^{-1}=T_{\theta},
$$

for a unique $0 \leqq \theta \leqq \pi / 3$.

Theorem 3.4. For $Q \neq 0, H(Q)$ is quaternionically simple.

Proof. For $r \in \mathbf{R}, H(r Q)=r H(Q)$, so it suffices to consider unit quadrupoles. By Proposition 3.3 it suffices to consider $T_{\theta}$, for $0 \leqq \theta \leqq \pi / 3$.

Use the basis of Definition 2.5 and the dictionary (2.17), (2.18) to write $T_{0}$ and $T_{3}$ as quaternion-valued matrices. $T_{0}=J_{3}^{2}-\mathbf{J}^{2} / 3$, so the corresponding quaternionic matrix is diagonal. Since the matrix is quaternionic hermitian, the diagonal entries must all be real. $T_{3}=(1 / \sqrt{3})\left(J_{1}^{2}-J_{2}^{2}\right)=(1 / \sqrt{3}) \frac{1}{2}\left(J_{+}^{2}+J_{-}^{2}\right)$. The only nonzero matrix elements are $\left\langle m \pm 1\left|T_{3}\right| m \mp 1\right\rangle$, none of which vanish for $-J+1 \leqq m \leqq J-1$. In the quaternionic basis, this takes the form of a real tridiagonal matrix with zeros on the diagonal, and no zeros on the subdiagonal or superdiagonal.

$T_{0}=J_{3}^{2}-\mathbf{J}^{2} / 3$ is readily seen to be quaternionically simple. For $0<\theta \leqq \pi / 3, T_{\theta}$ is a tridiagonal hermitian quaternionic matrix with no zeros on the subdiagonal and superdiagonal, and hence simple by Proposition 3.2.

It is noteworthy that the degeneracy structure of both $H(Q)$ and $H(\mathbf{B})$ is what one expects on the basis of the Wigner-von Neumann no crossing rule [23,24]. For the general (not time-reversal invariant) case, this says that degeneracies are codimension 3, and so isolated points in generic 3-spaces. Since $H(\mathbf{B})$ is homogeneous in $\mathbf{B}$, we may expect it to be simple for $\mathbf{B} \in \mathbf{R}^{3} /\{0\}$, and it is. For fermions with time reversal the codimension is five. So again by the homogeneity, $H(Q)$ we may expect it to be simple on $\mathbf{R}^{5} /\{0\}$, and it is.

3.3 A Child's Garden of Matricial Miscellanea. In this section we exhibit some properties of $H(Q)$ that are useful in the next chapter.

Proposition 3.5. For $Q$ and $Q^{\prime}$ unit quadrupoles, $\operatorname{Tr}(H(Q))=0$, and

$$
\operatorname{Tr}\left(H(Q) H\left(Q^{\prime}\right)\right)=K_{J} \operatorname{Tr}\left(Q Q^{\prime}\right) .
$$

It follows that

$$
\operatorname{Tr}\left(T_{\alpha} T_{\beta}\right)=K_{J} \delta_{\alpha, \beta},
$$

where the constant $K_{J}$ is independent of $\alpha$ and $\beta$.

Proof. The quadrupole operators $H(Q)$ are $J=2$ spherical tensor operators. $\operatorname{Tr}(H(Q))$ is a $J=0$ tensor. A linear function of $J=2$ tensors cannot be a nonzero 
$J=0$ tensor, so $\operatorname{Tr}(H(Q))=0$. Now $H(Q) H\left(Q^{\prime}\right)$ is the product of two $J=2$ tensors, and by the addition rules for angular momenta contains a unique $J=0$ tensor.

Along the lines of the proof of Theorem 3.4, we obtain results on the structure of the $T_{\alpha}$ as quaternionic matrices. These are summarized in:

Proposition 3.6. As quaternionic matrices in the basis of Definition 2.5

a) $T_{0}$ is real and diagonal;

b) $T_{\alpha}$, for $\alpha=1,2,3,4$ is zero on the diagonal;

c) All entries of $T_{1}\left(T_{2}, T_{3}, T_{4}\right)$ are real multiples of the unit quaternion $j,(k, 1, i$ respectively).

\section{The Homotopy of Simple Matrices}

We address here the computation of the homotopy groups of the simple hermitian matrices. Although the methods are standard, we present the calculation in detail, since it provides a context for one understanding of the Chern classes for the spectral bundles of Hamiltonians, and shows why the second Chern numbers are the only topological invariants for our quadrupole examples. Although most of the present work is concerned with the quaternionic case, it is convenient, for the purpose of this section, to consider this in parallel to the real and complex cases. An introduction for physicists to the relevant homotopy theory is given by Mermin [25]. Some of the material in this section recalls results in [26].

We distinguish two types of topological invariants: those associated to the space of matrices as a whole (global invariants), and those associated to individual parts of the spectrum (local invariants). Both global and local invariants can be integers $(\mathbf{Z})$ or torsion $\left(\mathbf{Z}_{p}=\mathbf{Z} / p \mathbf{Z}\right)$, or combinations of both. The Chern numbers are of course local in the above sense. Finally, the homotopy groups may also indicate the existence global sum rules satisfied by local invariants.

Definition 4.1. We denote by $M_{n}(\mathbf{R}), M_{n}(\mathbf{C})$, and $M_{n}(\mathbf{H})$ the real, complex, and quaternionic hermitian $n \times n$ matrices that are simple. $U_{n}(\mathbf{R}), U_{n}(\mathbf{C})$, and $U_{n}(\mathbf{H})$ denote the corresponding $n \times n$ unitary matrices. Thus

$$
U_{n}(\mathbf{R})=O(n), \quad U_{n}(\mathbf{C})=U(n), \quad U_{n}(\mathbf{H})=S p(n),
$$

in the standard notation.

Proposition 4.2. $M_{n}(\mathbf{F}) \quad(\mathbf{F}=\mathbf{R}, \mathbf{C}$, or $\mathbf{H})$ is path-connected. Equivalently, $\pi_{0}\left[M_{n}(\mathbf{F})\right]=0$, where 0 is the group (set) with one element.

Proof. Every hermitian matrix is diagonalizable by conjugation with a unitary matrix, preserving spectrum. The entries of a diagonal matrix can be arbitrarily permuted by conjugation with unitaries. Thus there exists for each simple hermitian matrix $A$ a unitary $U$ such that $U A U^{-1}$ is a diagonal matrix with the diagonal entries in decreasing order. This unitary can further be taken to be path-connected to the identity (for $\mathbf{F}=\mathbf{R}$, this means $U \in S O(n)$, and this imposes no restriction when $\mathbf{F}=\mathbf{C}, \mathbf{H}$.) The set $D_{n}$ of all such ordered diagonal matrices is convex, and 
hence contractible. Each simple hermitian matrix is thus path-connected to an element of $D_{n}$, and the result follows.

The basic tool in the computation of the higher homotopy groups of $M_{n}(\mathbf{F})$ is the identification of this space as a quotient:

Proposition 4.3. $M_{n}(\mathbf{F})$ is homotopic to the quotient $U_{n}(\mathbf{F}) /\left[U_{1}(\mathbf{F})\right]^{n}$.

Proof. A matrix in $M_{n}(\mathbf{F})$ is uniquely determined by its spectrum and by its eigenvectors. Fixing the spectrum is the same as choosing an element of $D_{n}$, the real ordered diagonal matrices. Fixing the eigenvectors is the same as choosing an element of $U_{n} /\left[U_{1}\right]^{n}$, since each eigenvector has a $U_{1}$ phase ambiguity. Thus

$$
M_{n}(\mathbf{F}) \simeq D_{n} \times U_{n}(\mathbf{F}) /\left[U_{1}(\mathbf{F})\right]^{n} .
$$

Since $D_{n}$ is contractible, the result follows.

This fact, together with the homotopy exact sequence, relate the homotopy groups of $M_{n}(\mathbf{F}), U_{n}(\mathbf{F})$, and $U_{1}(\mathbf{F}) . U_{1}(\mathbf{R})=O(1)$ is the 0 -sphere, $S^{0}=\{ \pm 1\}$; $U_{1}(\mathbf{C})=U(1)$ is the 1 -sphere $S^{1}$, and $U_{1}(\mathbf{H})=S p(1)$ is the 3 -sphere $S^{3}$. The homotopy groups of $S^{0}$ and $S^{1}$ are particularly simple, which makes the real and complex cases simpler than the quaternionic.

The basic result is: $[27,28]$

Theorem 4.4. There exists a natural exact sequence of group homomorphisms

$$
\cdots \rightarrow \pi_{m}\left[U_{1}(\mathbf{F})\right]^{n} \stackrel{\sigma_{m}}{\rightarrow} \pi_{m}\left[U_{n}(\mathbf{F})\right] \stackrel{\alpha_{m}}{\rightarrow} \pi_{m}\left[M_{n}(\mathbf{F})\right] \stackrel{\beta_{m}}{\rightarrow} \pi_{m-1}\left[\dot{U}_{1}(\mathbf{F})\right]^{n} \rightarrow \cdots .
$$

That is, the kernel of each map is the image of the preceding map.

The following basic results are useful for extracting information from this sequence:

\section{Lemma 4.5.}

a) If in an exact sequence an adjacent pair of groups is flanked by zeros, then the two groups are isomorphic. That is

$$
0 \stackrel{\beta}{\rightarrow} B \stackrel{\stackrel{\vartheta}{\rightarrow}}{\rightarrow} C \stackrel{\delta}{\rightarrow} 0 \quad \Rightarrow B \simeq C .
$$

b) If in the exact sequence

$$
\cdots \stackrel{\alpha}{\rightarrow} A \stackrel{\beta}{\rightarrow} B \stackrel{\vee}{\rightarrow} C \stackrel{\delta}{\rightarrow} D \rightarrow \cdots
$$

$\alpha$ is onto, then $B$ is isomorphic to the kernel of $\delta$.

c) In the exact sequence

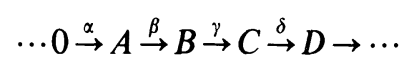

$\beta$ is $1-1$, and $B / A \simeq \operatorname{ker}(\delta)$.

Proof. A standard exercise in diagram chasing. 
For $S^{0}$ all the homotopy groups except $\pi_{0}$ are trivial. For $S^{1}$ all the homotopy groups except $\pi_{1}$ are trivial, and for $S^{3} \pi_{0,1,2}$ are trivial. Part a) of Lemma 4.5 together with the sequence (4.3) then give:

\section{Corollary 4.6.}

$$
\begin{array}{ll}
\pi_{m}\left[M_{n}(\mathbf{R})\right] \simeq \pi_{m}[O(n)], & m \geqq 2, \\
\pi_{m}\left[M_{n}(\mathbf{C})\right] \simeq \pi_{m}[U(n)], & m \geqq 3, \quad m=0, \\
\pi_{m}\left[M_{n}(\mathbf{H})\right] \simeq \pi_{m}[S p(n)], & m \leqq 2 .
\end{array}
$$

Since $\pi_{2}$ of the classical Lie groups is trivial, we see from (4.7) that $\pi_{2}\left[M_{n}(\mathbf{R})\right]$ and $\pi_{2}\left[M_{n}(\mathbf{H})\right]$ are both trivial. This is consistent with the vanishing of the first Chern class in time-reversal invariant cases, for both Bose and Fermi systems.

To proceed further we need to examine the group homomorphisms in (4.3) Define $f$ to be the real dimension of $\mathbf{F}, f=1,2,4$ for $\mathbf{F}=\mathbf{R}, \mathbf{C}, \mathbf{H}$ respectively.

Lemma 4.7. The inclusion $U_{1}(\mathbf{F}) \rightarrow U_{n}(\mathbf{F})$ induces an isomorphism of homotopy groups $\pi_{m}\left(U_{1}(\mathbf{F})\right) \rightarrow \pi_{m}\left(U_{n}(\mathbf{F})\right)$ for $m<2 f-2$.

Proof. Use the exact sequence of the fibration $U_{k}(\mathbf{F}) / U_{k-1}(\mathbf{F})=S^{k f-1}$. We assume the standard inclusion of $U_{k-1}$ into $U_{k}$ into the upper left corner and recall that the map $\sigma_{m}$ is the map induced by the inclusion [27]. Using $\pi_{m}\left(S^{k f-1}\right)=0$ for $m<k f-1$, and the long exact homotopy sequence for this fibration,

$$
\cdots \rightarrow \pi_{m}\left(U_{k-1}(\mathbf{F})\right) \stackrel{\rho_{m, k}}{\longrightarrow} \pi_{m}\left(U_{k}(\mathbf{F})\right) \rightarrow \pi_{m}\left(S^{k f-1}\right) \rightarrow \cdots,
$$

we find that the inclusion map $\rho_{m, k}$

$$
\pi_{m}\left(U_{k-1}(\mathbf{F})\right) \stackrel{\rho_{m, k}}{\longrightarrow} \pi_{m}\left(U_{k}(\mathbf{F})\right), \quad m<k f-2,
$$

is an isomorphism. It follows that the composition of inclusions

$$
\rho_{m, n} \circ \cdots \circ \rho_{m, 2}: \pi_{m}\left(U_{1}(\mathbf{F})\right) \rightarrow \pi_{m}\left(U_{n}(\mathbf{F})\right), \quad m<2 f-2<3 f-2 \cdots
$$

is an isomorphism.

\section{Lemma 4.8 .}

a) For $k<2 f-2$, the map $\sigma_{k}$ in (4.3) is the sum map:

$$
\begin{aligned}
& \sigma_{k}: \pi_{k}\left[U_{1}(\mathbf{F})\right]^{n} \rightarrow \pi_{k}\left[U_{n}(\mathbf{F})\right] \simeq \pi_{k}\left[U_{1}(\mathbf{F})\right] \\
& \left(z_{1}, \ldots, z_{n}\right) \mapsto z_{1}+\cdots+z_{n} .
\end{aligned}
$$

It is furthermore onto, and

$$
\operatorname{ker} \sigma_{k} \simeq \pi_{k}\left[U_{1}(\mathbf{F})\right]^{n-1}=\left[\pi_{k}\left(S^{f-1}\right)\right]^{n-1} .
$$

b) $\sigma_{2}$ is always an isomorphism.

Proof. a): From Lemma 4.7, we see that $\sigma_{m}\left(z_{1}, 0, \ldots, 0\right)=z_{1}$. From Lemma 4.7 and the group structure, we verify Eq. (4.11), and (4.12) follows. b): $\pi_{2}$ of the classical Lie groups is zero, so the map $\sigma_{2}: 0 \rightarrow 0$ is an isomorphism. 
We now have the main result of this section, which describes the homotopy groups associated to local topological invariants. The following theorem complements Corollary 4.6 and Proposition 4.2.

\section{Theorem 4.9.}

a) For $0 \leqq m \leqq 5$,

$$
\pi_{m}\left(M_{n}(\mathbf{H})\right)=\left[\pi_{m-1}(S p(1))\right]^{n-1} .
$$

In particular, $\pi_{m}\left(M_{n}(\mathbf{H})\right)=0$ for $m \leqq 3$, and

$$
\pi_{4}\left(M_{n}(\mathbf{H})\right)=\mathbf{Z}^{n-1}, \quad \pi_{5}\left(M_{n}(\mathbf{H})\right)=\mathbf{Z}_{2}^{n-1} .
$$

b) For $0 \leqq m \leqq 2$,

$$
\pi_{m}\left(M_{n}(\mathbf{C})=\left[\pi_{m-1}(U(1))\right]^{n-1} .\right.
$$

In particular, $\pi_{m}\left(M_{n}(C)\right)=0$ for $m \leqq 2$, and

$$
\pi_{2}\left(M_{n}(\mathbf{C})\right)=\mathbf{Z}^{n-1} \text {. }
$$

c) For $n \geqq 3$,

$$
\left[\pi_{1}\left(M_{n}(\mathbf{R})\right)\right] / \mathbf{Z}_{2}=\mathbf{Z}_{2}^{n-1} .
$$

The interpretation of this is as follows. The homotopy groups in (4.14) and (4.16) are those that give a topological invariant for each eigenvalue, constrained by one sum rule. There are no additional global invariants in these cases. ${ }^{6}$ The homotopy group in (4.17) is similar, except that there is an additional global $\mathbf{Z}_{2}$ invariant not associated to individual eigenvalues. For the real case the local invariant is in $\mathbf{Z}_{2}$, and is called the "Longuet-Higgins charge" for bosonic, time-reversal invariant situations [29]. It can be computed from the adiabatic holonomy as a line integral. It corresponds to the first Stiefel-Whitney number $w_{1}[30]$ of the eigenvalue bundle. $\pi_{2}\left[M_{n}(\mathbf{C})\right]$ corresponds to the $\mathrm{TKN}^{2}$ integers [31,32], or equivalently to the first Chern numbers [26]. $\pi_{4}\left[M_{n}(\mathbf{H})\right]$ corresponds to the second Chern numbers, our main concern here. Notice that time-reversal invariant fermions do not have a Longuet-Higgins charge associated to closed loops, since by Corollary 4.6 $\pi_{1}\left[M_{n}(\mathbf{H})\right]=0$. They do, however, have further local topological invariants, e.g., $\pi_{5}\left[M_{n}(\mathbf{H})\right]$, to be compared with the general local cohomological invariants discussed by Chang and Liang [33]. We note that for $\mathbf{F}=\mathbf{R}$ or $\mathbf{C}$, the higher invariants are global. ${ }^{7}$ We now prove the theorem:

Proof. a) follows from applying part b) of Lemma 4.5 to the long exact sequence (4.3). Define $\alpha=\sigma_{m}$ and $\delta=\sigma_{m-1}$, and use Lemma 4.8. Conclude from Lemma 4.5

\footnotetext{
${ }^{6}$ Recall that vector bundles over $S^{n}$ with fiber $\mathbf{F}$ are classified by $\pi_{n-1}\left(S^{f-1}\right)$ [27]. Real line bundles over $S^{n}$ are trivial for $n \geqq 2$, and complex line bundles are trivial for $n \geqq 3$. However, for any $n \geqq 4$, there exists nontrivial quaternionic line bundles over $S^{n}$

${ }^{7}$ This follows from the stability of the homotopy groups $\pi_{m}(U(n))$ and $\pi_{m}(O(n))$, i.e., for large enough $n$, the given homotopy group is independent of $n$, and from Corollary 4.6. Stability is an easy consequence of the exact sequence for $U_{k}(\mathbf{F}) / U_{k-1}(\mathbf{F})=S^{k S-1}$
} 
that $B=\pi_{m}\left(M_{n}(\mathbf{F})\right)$ is equal to the kernel of $\delta=\sigma_{m-1}$ to complete the proof. b) is similar. For $m=1$, the proof is identical to part a). For $m=2$, we use the additional fact that $\sigma_{2}$ is an isomorphism, and thus onto, and proceed as before. Part c) uses part c) of Lemma 4.5 , with $B=\pi_{1}\left(M_{n}(\mathbf{R})\right)$. We need the condition $n \geqq 3$ to ensure that $\pi_{1}(O(n))=\mathbf{Z}_{2}$.

\section{5. $J=3 / 2$ Quadrupole Hamiltonians}

We now discuss some features of $H(Q)$ which are particular to $J=3 / 2$.

5.1 SO(5) Symmetry. When is $H(Q)$ unitarily related to $H\left(Q^{\prime}\right)$ ? We shall find for $J=3 / 2$ all $H(Q)$ with $Q$ a unit quadrupole are unitarily related. In contrast, for $J>3 / 2$, there is a one dimensional family $\left\{T_{\theta}\right\} \subset\{H(Q)\}$ of unitarily inequivalent unit quadrupole Hamiltonians. Recall that for any $J$, Proposition 3.3 states that $H(Q)$ is unitarily related to $H\left(Q^{\prime}\right)$ if $Q$ and $Q^{\prime}$ are related by an $S O(3)$ rotation.

For $J=3 / 2$, the set of $H(Q)$ is precisely the set of all traceless quaternionic hermitian matrices. The set of $H(Q)$ for unit $Q$ is the set of traceless quaternionic hermitian matrices of unit norm, all of which are unitarily related.

We first show that this is not true for $J>3 / 2$.

Proposition 5.1. $T_{0}$ is unitarily related to $-T_{0}$ if and only if $J=3 / 2$ or $J=1 / 2$.

Proof. The largest eigenvalues of $T_{0}=J_{3}^{2}-\mathbf{J}^{2} / 3$ is equal to $J^{2}-J(J+1) / 3$. The largest eigenvalue of $-T_{0}=-J_{3}^{2}+\mathbf{J}^{2} / 3$ is equal to $-1 / 4+J(J+1) / 3$. Setting these equal gives a quadratic equation with solutions $J=1 / 2$ and $J=3 / 2$.

The $J=1 / 2$ case is trivial, $H(Q)=0$ for all $Q$.

In the remainder of this section, we shall examine symmetry properties of $H(Q)$ for $J=3 / 2$. This is all done in a basis-independent way. However, for reference we write below the matrices corresponding to $T_{\alpha}$ in the basis of Definition 2.5. Using Proposition 3.6, the matrices are determined up to overall real constants. The final result is:

$$
\begin{array}{ll}
T_{0}=\left(\begin{array}{cc}
1 & 0 \\
0 & -1
\end{array}\right), & \\
T_{1}=\left(\begin{array}{cc}
0 & -j \\
j & 0
\end{array}\right), & T_{2}=\left(\begin{array}{cc}
0 & k \\
-k & 0
\end{array}\right), \\
T_{3}=\left(\begin{array}{ll}
0 & 1 \\
1 & 0
\end{array}\right), & T_{4}=\left(\begin{array}{cc}
0 & -i \\
i & 0
\end{array}\right) .
\end{array}
$$

One checks by explicit matrix computation using (5.1) that

$$
T_{0} T_{1} T_{2} T_{3} T_{4}=-1 \text {. }
$$

We now examine the symmetry properties.

Lemma 5.2. For $J=3 / 2$,

$$
[H(Q)]^{2}=\frac{3}{2} \operatorname{Tr}\left(Q^{2}\right)
$$


Proof. For $Q \neq 0$, the sum of the two distinct eigenvalues of $H(Q)$ is zero by Proposition 3.5. $H(Q)^{2}$ is then a multiple of the identity operator, the normalization coming from Eq. (3.9).

The first consequence is:

Proposition 5.3. For $J=3 / 2$, and $Q$ and $Q^{\prime}$ any two unit quadrupoles, $H(Q)$ is unitarily related to $H\left(Q^{\prime}\right)$.

Proof. For $Q$ a unit quadrupole, Lemma 5.2 tells us that the squares of the eigenvalues are +1 . Since $H(Q)$ is traceless, the eigenvalues are $\{1,-1\}$. By the spectral theorem, all $H(Q)$ with unit $Q$ are unitarily (symplectically) related.

The space of quadrupole operators is a real five-dimensional vector space with inner product given by (3.9). There is thus an action of $S O(5)$ on this space. For general $J$, only an $S O(3)$ subgroup of this is a symmetry. For $J=3 / 2$, from Proposition 5.3, we see that $S O(5)$ is a symmetry. We now examine how this symmetry is implemented on $H(Q)$. For a rotation $G \in S O(5)$, we look for a unitary $U(G)$ such that

$$
H(G(Q))=U(G) H(Q) U(G)^{-1} .
$$

This is a generalization of Eq. (3.7) for the $S O(3)$ action. Recall that for $R \in S O(3)$, $U(R)$ is determined only up to a sign. It is also well known that $R \rightarrow U(R)$ is a representation of $S U(2)$, the two-fold cover of $S O(3)$, but only a projective representation of $S O(3)$. This is exactly what happens with the $S O(5)$ symmetry. There is no $S O(5)$ representation $G \rightarrow U(G)$ of the form above, but there is a representation of Spin (5), the two-fold cover of $S O(5)$. Equivalently, this is a projective representation of $S O(5)$.

The Spin(5) action is best understood through Clifford algebras.

Proposition 5.4. For $J=3 / 2$, the operators $H(Q)$ form a Clifford algebra:

$$
H(Q) H\left(Q^{\prime}\right)+H\left(Q^{\prime}\right) H(Q)=2 \frac{3}{2} \operatorname{Tr}\left(Q Q^{\prime}\right) .
$$

Equivalently, in the basis $T_{\alpha}$,

$$
T_{\alpha} T_{\beta}+T_{\beta} T_{\alpha}=2 \delta_{\alpha, \beta} .
$$

Proof. Apply Lemma 5.2 to $H\left(Q+Q^{\prime}\right)$.

$$
\begin{aligned}
{\left[H\left(Q+Q^{\prime}\right)\right]^{2} } & =\frac{3}{2} \operatorname{Tr}\left(Q+Q^{\prime}\right)^{2}, \\
H(Q) H\left(Q^{\prime}\right)+H\left(Q^{\prime}\right) H(Q) & =2 \frac{3}{2} \operatorname{Tr}\left(Q Q^{\prime}\right) .
\end{aligned}
$$

It is standard lore that the commutators $\left[H(Q), H\left(Q^{\prime}\right)\right]$, or $\left[T_{\alpha}, T_{\beta}\right], 0 \leqq \alpha<\beta \leqq 4$, span the ten-dimensional Lie algebra of the group Spin (5). The $H(Q)$ transform in the vector representation. This is the fundamental representation of $S O(5)$ on the space of quadrupoles.

It is well known that $\operatorname{Spin}(5) \simeq S p(2)$. The $S p(2)$ symmetry on our system can be readily understood directly. Note that the real dimension of the space $W$ of traceless quaternionic hermitian operators on $\mathbf{H}^{2}$ is five, the same as the dimension of the space $V$ of quadrupoles. 
Lemma 5.5. The map $V \rightarrow W$ which maps a quadrupole $Q$ to the operator $H(Q)$ is a linear isomorphism.

Proof. Since $H(Q)$ is simple for $Q \neq 0$, thus the kernel is 0 , and the map is one to one. The dimension of both vector spaces is the same, so an injection is an isomorphism.

It follows that all operators on $\mathbf{H}^{2}$ with spectrum $\{-1,+1\}$ are $H(Q)$ for some unit quadrupole $Q$. For $U \in S p(2), U H(Q) U^{-1}=H\left(Q^{\prime}\right)$. This gives an action of $S p(2)$ on the space of quadrupoles.

Finally, the generators of the rotation group form a subalgebra of the spin (5) Lie algebra. It useful for a later chapter to work these out explicitly in terms of the basis $T_{\alpha}$ of the Clifford algebra:

\section{Lemma 5.6.}

$$
\begin{aligned}
& -i J_{1}=\frac{1}{2}\left(\sqrt{3} T_{0} T_{2}+T_{1} T_{4}-T_{2} T_{3}\right), \\
& -i J_{2}=\frac{1}{2}\left(-\sqrt{3} T_{0} T_{1}-T_{1} T_{3}-T_{2} T_{4}\right) \\
& -i J_{3}=-\left(\frac{1}{2} T_{1} T_{2}+T_{3} T_{4}\right) .
\end{aligned}
$$

5.2 Chern Numbers. We now compute the Chern numbers of the $J=3 / 2$ spectral bundles.

Lemma 5.7. The spectral projections $P_{ \pm}$onto the positive and negative eigenspaces of $H(Q)$ for unit $Q$ are given by

$$
P_{ \pm}=\frac{1}{2}(1 \pm H(Q)) \text {. }
$$

Proof. By Lemma 5.2, $P_{ \pm}$are projections. $H(Q)=P_{+}-P_{-}$.

The $x_{\mu}$ are coordinates on the space of quadrupole operators, $H(Q)=x_{\mu} T_{\mu}$.

Lemma 5.8. At $T_{0}$,

$$
\left[\Omega_{ \pm}\right]^{2}=\mp \frac{3}{2} \frac{1}{2}\left(1 \pm T_{0}\right) d x_{1} d x_{2} d x_{3} d x_{4},
$$

and $\operatorname{Tr}\left(\Omega_{ \pm}^{2}\right)=\mp 3 d x_{1} d x_{2} d x_{3} d x_{4}$.

Proof. In general, $\left[P,(d P)^{2}\right]=0$, so $\Omega^{2}=P(d P)^{4}$. Substituting $(d P)= \pm \frac{1}{2} T_{\mu} d x_{\mu}$ and using (5.2) proves the first part. The trace (over $\mathbf{C}$ ) of the projection is equal to 2 .

The Chern numbers are now easily computed.

Proposition 5.9. The second Chern numbers for the spectral bundles of positive and negative energies are +1 and -1 respectively.

Proof. At $T_{0}$, we have by Definition 1.5,

$$
\begin{aligned}
\omega_{2}\left(P_{ \pm}\right) & =\frac{(-)^{2}}{(2 \pi i)^{2} 2 !}(\mp 3) d x_{1} d x_{2} d x_{3} d x_{4} \\
& = \pm \frac{3}{8 \pi^{2}} d x_{1} d x_{2} d x_{3} d x_{4} .
\end{aligned}
$$


Using the $S O(5)$ symmetry, we find $C_{2}\left(P_{ \pm}, S^{4}\right)= \pm 1$, since the volume of the unit sphere $S^{4}$ is equal to $8 \pi^{2} / 3$.

We finally note the duality properties of the curvature [34]. Let $*$ be the Hodge dual on forms $[8,34]$.

Proposition 5.10. The curvatures $\Omega_{+}$and $\Omega_{-}$are self-dual and anti-self-dual respectively everywhere on $S^{4}$ :

$$
* \Omega_{ \pm}= \pm \Omega_{ \pm}
$$

Proof. At $T_{0}$,

$$
\Omega_{ \pm}=\frac{1}{2}\left(1 \pm T_{0}\right) \frac{1}{4} T_{\mu} T_{v} d x_{\mu} d x_{v} .
$$

Compare for example the $d x_{1} d x_{2}$ component with the $d x_{3} d x_{4}$ component:

$$
\left[\Omega_{ \pm}\right]_{1,2}=\frac{1}{2}\left(1 \pm T_{0}\right) \frac{1}{2} T_{1} T_{2}= \pm \frac{1}{4}\left(T_{3} T_{4} \pm T_{1} T_{2}\right)= \pm\left[\Omega_{ \pm}\right]_{3,4} .
$$

We have used (5.2). The other components are analogous. The result for all of $S^{4}$ follows from the transitive action of the $S O(5)$ symmetry group.

Readers familiar with the $S U(2)$ Yang-Mills instanton or with canonical bundles over projective spaces will recognize these results as expressing the following abstract observations. By Lemma 5.5, the spectral bundles are identified with the canonical quaternionic line bundle over the quaternionic projective space $\mathbf{H} P^{1}$, which is homeomorphic to $S^{4}[35,34]$. The second Chern number of the canonical bundle is known to be +1 , and consequently the Chern numbers of the two spectral bundles are +1 and -1 [5]. We perform this standard computation by using the transitive symmetry to evaluate the integral of $\Omega^{2}$ in terms of the value at $T_{0}$. This bundle and connection are also known as the symmetric Yang-Mills instanton. We derive the known fact that the adiabatic curvature is self-dual. The self-duality of the adiabatic curvature for $J>3 / 2$ will be investigated in the next chapter.

\section{6. $J>3 / 2$ Quadrupole Hamiltonians}

In this chapter we compute $C_{2}$ of the spectral bundles of $H(Q)$ over the 4-sphere of unit quadrupoles for $J \geqq 3 / 2$. The Chern numbers are of course well defined, since we have already proved that all nonzero $H(Q)$ are simple. The method we use in this section is similar to that of Sect. 1.2, where we computed the first Chern numbers for Berry's example. Using Stokes' theorem, we reduce the integral of $\operatorname{Tr}\left(\Omega^{2}\right)$ over $S^{4}$ to the integral of a Chern-Simons form on 3-surfaces. Using the rotational symmetry, we then reduce these 3-dimensional integrals to traces of operators at the north and south poles, which we then evaluate. A variant of this calculation appeared in [22]. The Chern-Simons form and quaternions also appeared in a different context in [36].

6.1 The Chern-Simons Form. As in Sect. 1.2, we wish to find unitary operators $U(Q)$ such that $P(Q)=U(Q) P_{0} U(Q)^{-1}$, where $P_{0}$ is the spectral projection 
at the north pole. Then $\Omega=U\left(d \tilde{A}+\tilde{A}^{2}\right) U^{-1}$, where $\tilde{A}=P_{0} U^{-1}(d U) P_{0}$, as in Proposition 1.2, and we have

$$
\begin{aligned}
\operatorname{Tr}\left(\Omega^{2}\right) & =\operatorname{Tr}\left(\left[(d \tilde{A})+\tilde{A}^{2}\right]\left[(d \tilde{A})+\tilde{A}^{2}\right]\right) \\
& =\operatorname{Tr}\left[d(\tilde{A}(d \tilde{A}))+2 \tilde{A}^{2}(d \tilde{A})+\tilde{A}^{4}\right] .
\end{aligned}
$$

We choose $U(Q)$ as follows. Let $P_{\theta}$ be the projection associated with the Hamiltonian $T_{\theta}=T_{0} \cos (\theta)+T_{3} \sin (\theta)$, with $0 \leqq \theta \leqq \pi / 3$, and choose a unitary $V_{\theta}$ such that $P_{\theta}=V_{\theta} P_{0} V_{\theta}^{-1}$. By Proposition 3.3, we can take $U=W V$, where $W$ is a unitary belonging to the $S U(2)$ representation.

We remark that $U$ is only locally defined, and even then only on $S^{4} / N$, where $N \subset S^{4}$ is the set consisting of the two rotation group orbits that are two-dimensional. We recall that the north pole is contained in one of these orbits, and the south pole in the other. On the orbit of the north pole there are an infinite number of rotations $W$ that link $P_{0}$ to $P_{Q}$, since all rotations of the form $\exp \left(i \phi J_{3}\right)$ commute with $P_{0}$. This prevents a consistent choice of $W$, and so $U$ is ill defined. A similar problem exists at the south pole. For $Q \in S^{4} / N$, there is an eight-fold ambiguity in the choice of $W$, and hence of $U$, recall part c) of Proposition 3.1. ${ }^{8}$ Thus $\tilde{A}$ is only locally single-valued on $S^{4} / N$.

Noting that $\operatorname{Tr}\left(\tilde{A}^{4}\right)=\operatorname{Tr}\left(\tilde{A} \wedge \tilde{A}^{3}\right)=\operatorname{Tr}\left(-\tilde{A}^{3} \wedge \tilde{A}\right)=0,(6.1)$ gives

Lemma 6.1. On $S^{4} / N$, the second Chern form is given by

$$
\omega_{2}=\frac{-1}{8 \pi^{2}} \operatorname{Tr}\left(\Omega^{2}\right)=\frac{-1}{8 \pi^{2}} d S,
$$

where $S$ is the Chern-Simons 3-forms [37]

$$
S=\operatorname{Tr}\left[\tilde{A}(d \tilde{A})+\frac{2}{3} \tilde{A}^{3}\right] .
$$

Both $S$ and the second Chern form are single-valued on $S^{4} / N$, even though $\tilde{A}$ is multiple valued. The global ambiguity disappears when we take the trace. We can now use Stokes' theorem to obtain:

\section{Lemma 6.2.}

$$
C_{2}=\int_{S^{4}} \omega_{2}=\int_{S^{4} / N} \omega_{2}=\frac{-1}{8 \pi^{2}} \lim _{\varepsilon \rightarrow 0}\left(\int_{Y_{\pi / 3-\varepsilon}} S-\int_{Y_{\varepsilon}} S\right),
$$

where $Y_{\theta}$ is the orbit containing $T_{\theta}$.

6.2 Chern Numbers. We now integrate $S$ over $Y_{\theta}$. We first note that, restricted to $Y_{\theta}, d V=0$, and so

$$
\begin{aligned}
\tilde{A} & =P_{0} U^{-1}(d U) P_{0}=P_{0} V^{-1} W^{-1}(d W) V P_{0}=V^{-1} P_{\theta} W^{-1}(d W) P_{\theta} V \\
d \tilde{A} & =V^{-1} P_{\theta}\left(d W^{-1}\right)(d W) P_{\theta} V=-V^{-1} P_{\theta} W^{-1}(d W) W^{-1}(d W) P_{\theta} V
\end{aligned}
$$

\footnotetext{
${ }^{8}$ In the quaternionic basis of Definition 2.5, the eight element group consists of the diagonal matrices with entries $\pm 1, \pm i, \pm j$, and $\pm k$. These commute with real matrices, such as $P_{\theta}$
} 
Let $S_{\theta}$ be $S$ restricted to $Y_{\theta}$. Defining $\gamma=W^{-1}(d W)$, we then have

$$
S_{\theta}=\operatorname{Tr}\left[-P_{\theta} \gamma P_{\theta} \gamma^{2} P_{\theta}+\frac{2}{3}\left(P_{\theta} \gamma\right)^{3}\right] \text {. }
$$

$S_{\theta}$ is rotationally invariant. There is up to normalization a unique rotationally invariant three-form on $Y_{\theta}$, so we need merely determine this normalization. Examine $S_{\theta}$ at the origin, $W=1$. Near the origin we use the coordinates $\left\{y_{\alpha}\right\}$, $\alpha=1,2,3$, with $W=\exp \left(-i J_{\alpha} y_{\alpha}\right)$. This gives

$$
\begin{aligned}
\gamma= & -i J_{\alpha} d y_{\alpha}, \\
\gamma^{2}= & -i\left(J_{1} d y_{2} d y_{3}+J_{2} d y_{3} d y_{1}+J_{3} d y_{1} d y_{2}\right), \\
S_{\theta}= & \left(\operatorname{Tr}\left[P_{\theta} J_{1} P_{\theta} J_{1}+P_{\theta} J_{2} P_{\theta} J_{2}+P_{\theta} J_{3} P_{\theta} J_{3}\right]\right. \\
& \left.+2 i \operatorname{Tr}\left[P_{\theta} J_{1} P_{\theta} J_{2} P_{\theta} J_{3}-P_{\theta} J_{3} P_{\theta} J_{2} P_{\theta} J_{1}\right]\right) d y_{1} d y_{2} d y_{3} \\
\equiv & f\left(P_{\theta}\right) d y_{1} d y_{2} d y_{3} .
\end{aligned}
$$

The volume form $d y_{1} d y_{2} d y_{3}$ is independent of $\theta$, of $J$, and of the eigenvalue, so integrating $S$ over $Y_{\theta}$ just gives a constant times $f\left(P_{\theta}\right)$. Thus

$$
C_{2}=A\left[f\left(P_{0}\right)-f\left(P_{\pi / 3}\right)\right]=A\left[f\left(P_{0}\right)-f\left(P_{\pi}\right)\right],
$$

where $A$ is some normalization constant. The last equality comes from the fact that $P_{\pi / 3}$ and $P_{\pi}$ are rotationally related and $f$ is a rotationally invariant function. By knowing $C_{2}$ for $J=3 / 2$ we will fix $A$.

The spectrum of $H(Q)$ is labeled by the $J_{3}$ eigenvalue, as we now explain. Since there are no level crossing for $Q \neq 0$, each level of $H(Q)$ can be uniquely identified with a level of $T_{0}=J_{3}^{2}-\mathbf{J}^{2} / 3$. Each eigenspace of $T_{0}$ decomposes as the sum of two eigenspaces of $J_{3}$ with eigenvalues $\left\{m_{T},-m_{T}\right\}$. We thus label the spectrum of $H(Q)$ by the corresponding $m_{T}>0$. Similarly, the levels of $H(Q)$ can be identified with the levels of $-T_{0}=T_{\pi}$, yielding an analogous labeling which we denote by $m_{B}$. These are related by

We then have:

$$
m_{T}+m_{B}=J+\frac{1}{2}
$$

Theorem 6.3. The second Chern number $C_{2}\left(P_{m_{T}, J}, S^{4}\right)$ of the spectral bundle corresponding to the $m_{T}$ level of the spin $J$ quadrupole Hamiltonians $\{H(Q)\}$ is equal to

$$
C_{2}\left(P_{m_{T}, J} S^{4}\right)=\frac{m_{T}^{2}-m_{B}^{2}}{2}=\frac{\left(J+\frac{1}{2}\right)\left(2 m_{T}-J-\frac{1}{2}\right)}{2} .
$$

Proof. We calculate $f(P)$, for $P$ of the form $P=|J, m\rangle\langle J, m|+| J,-m\rangle\langle J,-m|$ with $m>0 . P_{0}$ is of this form with $m=m_{T}$, as is $P_{\pi}$ with $m=m_{B}$.

For $m>\frac{1}{2}$ we have $P J_{1} P=P J_{2} P=0$, and so $f(P)=\operatorname{Tr}\left(P J_{3} P J_{3}\right)=2 m^{2}$. For $m=\frac{1}{2}$, on the vector space with basis $\left|J, \frac{1}{2}\right\rangle,\left|J,-\frac{1}{2}\right\rangle$, we have $P J_{1} P=c \sigma_{1}$, $P J_{2} P=c \sigma_{2}$, and $P J_{3} P=\frac{1}{2} \sigma_{3}$, with $c=\left(J+\frac{1}{2}\right) / 2$. This gives

$$
\begin{array}{r}
\operatorname{Tr}\left(2 i P J_{1} P J_{2} P J_{3}\right)=\operatorname{Tr}\left(-2 i P J_{3} P J_{2} P J_{1}\right)=-2 c^{2} \\
\operatorname{Tr}\left(P J_{1} P J_{1}\right)=\operatorname{Tr}\left(P J_{2} P J_{2}\right)=2 c^{2} \\
\operatorname{Tr}\left(P J_{3} P J_{3}\right)=\frac{1}{2}=2 m^{2} .
\end{array}
$$


So $f(P)=2 m^{2}$ for all $m$.

Since $f\left(P_{0}\right)=2 m_{T}^{2}$ and $f\left(P_{\pi}\right)=2 m_{B}^{2}$, we have $C_{2}=2 A\left(m_{T}^{2}-m_{B}^{2}\right)$. For $J=3 / 2$, $m_{T}=3 / 2$ and $\left(m_{B}=\frac{1}{2}\right)$ we already know that $C_{2}=1$, implying that $A=1 / 4$ in Eq. (6.8).

6.3 Properties of the Adiabatic Connections. From Theorem 6.3 we see that different combinations of $J$ and $m_{T}$ may lead to the same Chern number $C_{2}$. For example, both the $\left(J=5 / 2, m_{T}=5 / 2\right)$ and the $\left(J=11 / 2, m_{T}=7 / 2\right)$ systems have $C_{2}=3$. The question naturally arises as whether distinct bundles with the same Chern number can have gauge-equivalent connections. We shall see that they cannot. We also consider whether any bundles have self-dual or anti-self-dual curvatures, and will see that this cannot occur for $J>3 / 2$.

Our basic tool is to examine the curvature at the north and south poles, which may be easily calculated using first order perturbation theory and Eq. (1.9). Using the coordinates $\left\{x_{\alpha}\right\} \leftrightarrow x_{\alpha} T_{\alpha}$ we have

Lemma 6.4. a) For $m_{T}>3 / 2$, the curvature at the north pole is given by

$$
\Omega=\frac{i m_{T}}{3} R\left[d x_{1} \wedge d x_{2}+\frac{K^{2}-\left(m_{T}^{2}-1\right)^{2}}{8\left(m_{T}^{2}-1\right)^{2}} d x_{3} \wedge d x_{4}\right],
$$

where $K \equiv J(J+1)$, and $R \equiv\left(\left|m_{T}\right\rangle\left\langle m_{T}|-| m_{T}\right\rangle\left\langle-m_{T}\right|\right)$.

b) For $m_{T}=3 / 2$, the curvature equals the one above, plus

$$
G\left|\frac{3}{2}\right\rangle\left\langle-\frac{3}{2}\left|\left(d x_{1}-i d x_{2}\right) \wedge\left(d x_{3}-i d x_{4}\right)-G\right|-\frac{3}{2}\right\rangle\left\langle\frac{3}{2}\right|\left(d x_{1}+i d x_{2}\right) \wedge\left(d x_{3}+i d x_{4}\right),
$$

where $G \equiv(J-1 / 2)(J+1 / 2)(J+3 / 2)$. In particular, for $m_{T}=3 / 2$, the $d x_{1} \wedge d x_{2}$ and $d x_{3} \wedge d x_{4}$ components are given by the same formulas as for $m_{T}>3 / 2$.

c) For $m_{T}=\frac{1}{2}$, the curvature is given by

$$
\begin{aligned}
\Omega_{1 / 2}= & \frac{i}{6}\left(\left|\frac{1}{2}\right\rangle\left\langle\frac{1}{2}|-|-\frac{1}{2}\right\rangle\left\langle-\frac{1}{2}\right|\right)\left[\left(\frac{3}{4}-K\right) d x_{1} \wedge d x_{2}+\frac{K^{2}-(3 / 4)^{2}}{8(3 / 4)^{2}} d x_{3} \wedge d x_{4}\right] \\
& +G\left|\frac{1}{2}\right\rangle\left\langle-\frac{1}{2}\right|\left(d x_{1}+i d x_{2}\right) \wedge\left(d x_{3}-i d x_{4}\right) \\
& -G\left|-\frac{1}{2}\right\rangle\left\langle\frac{1}{2}\right|\left(d x_{1}-i d x_{2}\right) \wedge\left(d x_{3}+i d x_{4}\right) .
\end{aligned}
$$

We remark that at the south pole the same formulas apply, only with $m_{T}$ replaced by $m_{B}=J+\frac{1}{2}-m_{T}$. The reason is that the energy levels, the eigenstates, and $d H$ are the same at the north and south poles, as are the tangent planes. The only difference between the two poles is that $d x_{1} \wedge d x_{2} \wedge d x_{3} \wedge d x_{4}$ is in the positive orientation at the north pole, but is in the negative orientation at the south pole, a distinction that has relevance to the question of self-duality.

Theorem 6.5. Suppose that the $\left(J, m_{T}\right)$ and the $\left(J^{\prime}, m_{T}^{\prime}\right)$ systems have the same Chern number and suppose that $m_{T}>m_{T}^{\prime}$. Then the two systems do not have gauge-equivalent connections.

Proof. If two connections do not have unitarily related curvatures at one point, 
then they cannot be gauge-equivalent. We examine the curvatures at the north pole. Note that the theorem covers all cases, since by (6.10) $m_{T}=m_{T}^{\prime}$ would imply $J=J^{\prime}$, and the systems would not be distinct. The proof is by cases:

i) If $m_{T}>m_{T}^{\prime}>3 / 2$, then the two curvatures differ in their $d x_{1} \wedge d x_{2}$ terms at the north pole. By Eq. (6.12), the magnitude of this term is proportional to $m_{T}$ and is independent of $J$.

ii) If $m_{T}>3 / 2 \geqq m_{T}^{\prime}$, then the curvature of the $\left(J^{\prime}, m_{T}^{\prime}\right)$ system at the north pole has a $d x_{1} \wedge d x_{3}$ terms, while the curvature of the $\left(J, m_{T}\right)$ system does not.

iii) If $m_{T}=3 / 2$ and $m_{T}^{\prime}=1 / 2$, we look at the magnitude of the $d x_{1} \wedge d x_{3}$ terms. These are equal to $(J-1 / 2)(J+1 / 2)(J+3 / 2)$ in one case and $\left(J^{\prime}-1 / 2\right)\left(J^{\prime}+1 / 2\right)$ $\left(J^{\prime}+3 / 2\right)$ in the other case. Since $J \neq J^{\prime}$, these magnitudes are different.

The other result of this section concerns self-duality.

Theorem 6.6. For $J>3 / 2$ the curvature $\Omega$ is never self-dual or anti-self dual.

Proof. We consider some necessary conditions for a curvature to be self-dual. At the north pole $*\left(d x_{1} \wedge d x_{2}\right)=d x_{3} \wedge d x_{4}$, so a self-dual curvature must have the same $d x_{1} \wedge d x_{2}$ and $d x_{3} \wedge d x_{4}$ terms at the north pole. This gives the condition

$$
\text { either } K^{2}-\left(m_{T}^{2}-1\right)^{2}=8\left(m_{T}^{2}-1\right)^{2} \text { or } m_{T}=1 / 2 \text {. }
$$

At the south pole $*\left(d x_{1} \wedge d x_{2}\right)=-d x_{3} \wedge d x_{4}$ so our condition there is

$$
\text { either } K^{2}-\left(m_{B}^{2}-1\right)^{2}=-8\left(m_{B}^{2}-1\right)^{2} \text { or } m_{B}=1 / 2 \text {. }
$$

The first equality in $(\mathrm{C} 2)$ can never be met, since the left-hand-side is positive and the right-hand-side is negative. Thus we must have $m_{B}=1 / 2$, which in turn implies $m_{T}=J$. Plugging this into $(\mathrm{C} 1)$ we get that either $J=\frac{1}{2}$, or $J(J+1) \equiv K=3\left(J^{2}-1\right)$, implying $J=3 / 2$. Thus the only possible self-dual system has $J=m_{T}=3 / 2$ or $J=m_{T}=\frac{1}{2}$. By a similar argument, the only possible anti-self-dual systems have $J=m_{B}=3 / 2$ (and so $m_{T}=1 / 2$ ) or $J=m_{B}=\frac{1}{2}$ (and so $m_{T}=\frac{1}{2}$ ).

For $J \leqq 3 / 2, \Omega$ is always either self-dual or anti-self-dual.

\section{Holonomy of the Adiabatic Connection}

The physical time evolution of a system, that generated by the Schrödinger equation, is in the adiabatic limit the same as the holonomy of the adiabatic connection discussed in Sect. 1.1. We now compute this holonomy for certain time-dependent quadrupole Hamiltonians. Holonomy calculations for other systems also appear in $[38,39,4,40,12]$. There is an explicit form of the solution of the adiabatic connection equation that will prove useful.

Proposition 7.1. $([2,3])$ Let $c(t)$ be a path on parameter space, and $P(t)=P(c(t))$. Define

$$
U_{n}(t)=P(t) P\left(\frac{(n-1) t}{n}\right) \cdots P\left(\frac{2 t}{n}\right) P\left(\frac{t}{n}\right) P(0) .
$$


Then

$$
U(t) P(0)=\lim _{n \rightarrow \infty} U_{n}(t)
$$

is a solution of the adiabatic evolution Eq. (1.6), with the initial state in the range of $P(0)$.

We shall in this section frequently write quaternionic matrices corresponding to operators using the basis of Definition 2.5 without further comment.

7.1 Symmetry Group Orbits. We now calculate the adiabatic time evolution for paths on the four-sphere of unit quadrupoles of the form

$$
T(t)=\exp (t A) T(0) \exp (-t A),
$$

where $A$ is an antihermitian generator of the symmetry group, which is $\operatorname{Spin}(3) \cong$ $S U(2)$ for $J>3 / 2$, and $\operatorname{Spin}(5) \cong S p(2)$ for $J=3 / 2$. Since the symmetry group preserves time-reversal invariance, $A$ necessarily satisfies $\Theta A \Theta^{-1}=A$. The generators of the $S U(2)$ symmetry are of the form $A=-i J_{\mu}$, and the generators of the Spin (5) symmetry of the form $A=\left[T_{\alpha}, T_{\beta}\right]$.

The spectral projection of $T(t)$ is given by

$$
P(t)=\exp (t A) P \exp (-t A),
$$

where $P=P(0)$. Use Proposition 7.1 to obtain the adiabatic evolution:

$$
U(t) P=\lim _{n \rightarrow \infty}\left[\exp \left(\frac{t}{n} A\right)\right]^{n}\left[P \exp \left(\frac{-t}{n} A\right) P\right]^{n} P=\exp (t A) \exp (-t P A P) P .
$$

Lemma 7.2. Let $P$ be a quaternionic projection of rank one, and $|\cdot|$ the quaternionic operator norm. If $A$ is an antihermitian quaternionic operator, then

$$
(P A P)^{2}=-|P A P|^{2} P .
$$

Proof. $(P A P)^{2}=-P \bar{A} P A P=-\overline{P A P} P A P$ is a negative hermitian operator of rank one. A hermitian quaternionic operator on $\mathbf{H}^{1}$ is a real multiple of the identity, and the norm of a projection is one. The result follows from applying the operator norm.

Lemma 7.3. Let $P$ and $A$ be as above. Then

$$
\exp (-t P A P)=\cos (t|P A P|) P-\frac{\sin (t|P A P|)}{|P A P|} P A P .
$$

Proof. Use the series expansion of the exponential function, and Lemma 7.2.

We finally obtain

\section{Proposition 7.4.}

$$
U(t) P=\exp (t A)\left[\cos (t|P A P|) P-P A P \frac{\sin (t|P A P|)}{|P A P|}\right] .
$$

We first examine $J=3 / 2$. Since there is a transitive symmetry group, we can without loss of generality consider paths starting at $T_{0}$. The antihermitian 
generators $A$ are linear combinations of $\frac{1}{2}\left[T_{\alpha}, T_{\beta}\right]=T_{\alpha} T_{\beta}, \alpha<\beta$. For $T_{0}$, the spectral projections onto the positive and negative eigenstates are

Define

$$
P_{ \pm}=\frac{1}{2}\left(1 \pm T_{0}\right)
$$

$$
\begin{aligned}
& \rho_{ \pm 1} \equiv \frac{1}{2}\left(T_{2} T_{3} \pm T_{1} T_{4}\right), \\
& \rho_{ \pm 2} \equiv \frac{1}{2}\left(-T_{1} T_{3} \pm T_{2} T_{4}\right), \\
& \rho_{ \pm 3} \equiv \frac{1}{2}\left(T_{3} T_{4} \pm T_{1} T_{2}\right) .
\end{aligned}
$$

In the basis (2.26), using (5.1),

$$
\begin{aligned}
& \rho_{+1}=\left(\begin{array}{ll}
k & 0 \\
0 & 0
\end{array}\right), \quad \rho_{+2}=\left(\begin{array}{ll}
j & 0 \\
0 & 0
\end{array}\right), \quad \rho_{+3}=\left(\begin{array}{ll}
i & 0 \\
0 & 0
\end{array}\right), \\
& \rho_{-1}=\left(\begin{array}{rr}
0 & 0 \\
0 & -k
\end{array}\right), \quad \rho_{-2}=\left(\begin{array}{rr}
0 & 0 \\
0 & -j
\end{array}\right), \quad \rho_{-3}=\left(\begin{array}{rr}
0 & 0 \\
0 & -i
\end{array}\right) .
\end{aligned}
$$

The following lemma easily follows from the expression (7.11), although we give an independent proof.

\section{Lemma 7.5.}

a)

b)

$$
P_{ \pm} T_{0} T_{\alpha} P_{ \pm}=0
$$

$$
\begin{aligned}
& P_{ \pm} \rho_{ \pm n} P_{ \pm}=\rho_{ \pm n} . \\
& P_{ \pm} \rho_{\mp n} P_{\mp}=0
\end{aligned}
$$

Proof. a) follows from the Clifford property of the $T_{\alpha}$. b) comes from $T_{0} T_{1} T_{2} T_{3} T_{4}=$ -1 , as in the proof of Lemma 7.6. It follows that $\rho_{+_{n}} \rho_{-m}=0$.

We remark that $\left\{\rho_{+n}\right\}$ and $\left\{\rho_{-n}\right\}$ each span a copy of the Lie algebra $s p(1) \cong$ spin (3). This can be understood by noting that a) shows that only the spin(4) subalgebra of spin(5) which leaves $T_{0}$ invariant is nonzero in $P A P{ }^{9}$

To demonstrate the technique, we present two examples:

Example 7.7. Let $A=-i\left(c J_{3}+s J_{1}\right)$, with $c^{2}+s^{2}=1$. Then $\exp (2 \pi A)=-1$, which leaves all the quadrupoles fixed, i.e,

$$
\exp (t A) T_{0} \exp (-t A), \quad 0 \leqq t \leqq 2 \pi
$$

is a closed path on the space of unit quadrupoles. Using (5.8) and Lemma 7.5,

$$
\begin{aligned}
P_{+} A P_{+} & =(-3 c / 2) \rho_{+3}, \\
\left|P_{+} A P_{+}\right| & =3 c / 2, \\
P_{-} A P_{-} & =(-c / 2) \rho_{-3}-s \rho_{-1}, \\
\left|P_{-} A P_{-}\right| & =\frac{1}{2} \sqrt{1+3 s^{2}} .
\end{aligned}
$$

${ }^{9}$ It is well known that $\operatorname{spin}(4) \cong \operatorname{spin}(3) \oplus \operatorname{Spin}(3)$ 
Using Proposition 7.4, the adiabatic time evolution operator for the upper two levels is

$$
\begin{aligned}
U(2 \pi) P_{+} & =(-)\left[\cos (3 \pi c) P_{+}+\sin (3 \pi c) \rho_{+3}\right]=-\exp (3 \pi c i), \\
U(2 \pi) P_{-} & =(-)\left[\cos \left(\pi \sqrt{1+3 s^{2}}\right) P_{-}+\frac{\sin \left(\pi \sqrt{1+3 s^{2}}\right)}{\sqrt{1+3 s^{2}}}\left(c \rho_{-3}+2 s \rho_{-1}\right)\right] \\
& =-\exp (-\pi(c i+2 s j)) .
\end{aligned}
$$

The equation for the upper level is precisely Berry's phase for $m=3 / 2$. The equation for the lower level gives a holonomy which has a $j$ component as well as $i$, and is not just a complex phase multiplying the function, but rather induces transitions between the $J_{3}= \pm \frac{1}{2}$ states. This formula has been derived in the papers $[4,40]$.

Example 7.8. By allowing $S O(5)$ rotations which do not lie in the subgroup of physical rotations, we can obtain any desired holonomy. As an example, take $A=\frac{1}{2} T_{1}\left(c T_{0}+s T_{3}\right)$, with $c^{2}+s^{2}=1$. Then $\exp (2 \pi A)=-1$, and

$$
\exp (t A) T_{0} \exp (-t A), \quad 0 \leqq t \leqq 2 \pi
$$

is a closed path on the space of unit quadrupoles.

$$
\begin{aligned}
P_{+} A P_{+} & =-\frac{1}{2} s \rho_{+2}, \\
\left|P_{+} A P_{+}\right| & =\frac{1}{2} s, \\
U(2 \pi) P_{+} & =(-)\left[\cos (\pi s)+\sin (\pi s) \rho_{+2}\right]=-\exp (\pi s j) .
\end{aligned}
$$

For $s=\frac{1}{2}, U(2 \pi) P_{+}=\rho_{+2}$. The holonomy is $j$, in other words, the adiabatic time evolution interchanges the $J_{3}= \pm 3 / 2$ eigenstates.

For $J>3 / 2$, the calculations are similar but more lengthy, and will not be performed in detail. The symmetry group is now $S U(2)$, which does not act transitively on $S^{4}$, As we saw earlier, we can without loss of generality consider paths starting at $T_{\theta}=\cos (\theta) T_{0}+\sin (\theta) T_{3}, 0 \leqq \theta \leqq \pi / 3$. The main difficulty comes in obtaining the spectral projections of $T_{\theta}$. For $J=5 / 2$, this is done in the appendix.

7.2 Split Spheres. The holonomy of a path can be easily found when the holonomy group of a connection is Abelian. We now look for subsets $M \subset H(Q)$ such that the adiabatic connection restricted to $M$ has Abelian holonomy group.

We shall study connections which arise from splitting projections.

Definition 7.9. A splitting projection is a complex hermitian projection $L$ that satisfies

$$
L+\Theta L \Theta^{-1}=1 .
$$

To each splitting projection $L$, we associate the set $M_{L}$ of $L$-split quadrupoles, the set of $H(Q)$ commuting with $L$. It is clear that $M_{L}$ is a vector subspace of $H(Q)$.

Proposition 7.10. $L$ splits the spectral bundles over $M_{L}$ as the sum of two complex line bundles, and the adiabatic time evolution respects the splitting, i.e.,

$$
U(t) L=L U(t) .
$$


Proof. For every $T \in M_{L}$, the spectral projections of $T$ commute with $L$. Since $T$ is time-reversal invariant, the spectral projections satisfy $\Theta P=P \Theta$. It follows that

$$
P=L P+\Theta L P \Theta^{-1},
$$

and $L P$ and $\Theta L P \Theta^{-1}$ are projections onto orthogonal subspaces, both of complex rank one. Let $c(t)$ be a path on $M_{L}$. Using Proposition 7.1, we obtain a solution of the adiabatic time evolution operator as the limit of a product of spectral projections;

$$
U(t)=\lim _{n \rightarrow \infty} P(t) P\left(\frac{(n-1) t}{n}\right) \cdots P\left(\frac{2 t}{n}\right) P\left(\frac{t}{n}\right) P(0) .
$$

Each of the projections $P(k t / n)$ commutes with $L$, proving Eq. (7.20).

We first show that the dimension of $M_{L}$ must be less than five: i.e., $M_{L}$ cannot be all of $H(Q)$.

Lemma 7.11. Let $F$ be a complex vector bundle of rank two over $S^{4}$. If $C_{2}(F) \neq 0$, then $F$ cannot be split as the sum of two complex line bundles.

Proof. Suppose that $F$ is the sum of two complex line bundles, $F=F_{1} \oplus F_{2}$. Since $H^{2}\left(S^{4}\right)=0, C_{1}\left(F_{i}\right)=0$. The Chern classes satisfy [30]

$$
C(F) \equiv 1+C_{1}(F)+C_{2}(F)=\left(1+C_{1}\left(F_{1}\right)\right)\left(1+C_{1}\left(F_{2}\right)\right),
$$

so if $F=F_{1} \oplus F_{2}$ then $C_{2}(F)=0$. By Theorem 6.3, $C_{2}$ of the spectral bundles is nonzero except for the middle eigenstate of the $J=1 / 2,5 / 2,9 / 2 \ldots$ Hamiltonians.

We now construct splitting projections from the symmetry group of $H(Q)$. The dimension of $M_{L}$ for these splitting projections is three. The intersection of the unit quadrupoles with $M_{L}$ is a two-sphere. There is a first Chern number associated with the split pair of bundles. In the following, all operators are considered as complex time-reversal invariant, rather than quaternionic. The difference is that $i A$ is a well-defined operator in the former sense, but not in the latter.

Lemma 7.12. Define

Then

$$
V_{L} \equiv i(1-2 L)
$$

a) the following are equivalent:

i) L is a splitting projection;

ii) $V_{L}$ is a time-reversal invariant unitary, and $V_{L}^{\dagger}=-V_{L}$.

b) For any complex-linear operator $A,[A, L]=0$ if and only if $V_{L} A V_{L}^{\dagger}=A$.

Proof. i) $\Rightarrow$ ii): $\Theta V_{L} \Theta^{-1}=-i(1-2(1-L))=V_{L} . V_{L}^{\dagger}=-i(1-2 L)=-V_{L}$, and the unitarity comes from $V_{L}^{\dagger} V_{L}=-i(1-2 L) i(1-2 L)=1$.

ii) $\Rightarrow \mathrm{i})$ : Inverting (7.24), $L=\frac{1}{2}\left(1+i V_{L}\right) . \quad L$ is hermitian, $L^{\dagger}=\frac{1}{2}\left(1-i V_{L}^{\dagger}\right)=L$. $\Theta L \Theta^{-1}=\frac{1}{2}\left(1-i V_{L}\right)=1-L$.

b): If $[A, L]=0$, then $\left[A, V_{L}\right]=0$, and $V_{L} A V_{L}^{\dagger}=A$ because $V_{L} V_{L}^{\dagger}=1$. To prove 
the converse, $V_{L} A V_{L}^{\dagger}=A$ is the same as $\left[V_{L}, A\right]=0$, and the result follows.

We first examine $J=3 / 2$. All time-reversal invariant unitaries satisfying $V^{\dagger}=-V$ have spectrum $\{i,-i\}$, and are related by a time-reversal invariant unitary, i.e., by an element of the $S p(2)$ symmetry group. We can without loss of generality examine just one such $V$, and the corresponding $L$.

Proposition 7.23. The operator $L$ defined by

$$
V_{L}=T_{1} T_{2}=i(1-2 L)
$$

is a splitting projection. The set $M_{L}$ of $H(Q)$ commuting with $L$ is the linear span of $\left\{T_{0}, T_{3}, T_{4}\right\}$.

Proof. $\left(T_{1} T_{2}\right)^{\dagger}=T_{2} T_{1}=-T_{1} T_{2}$, and $T_{1} T_{2}$ is clearly time-reversal invariant. By Lemma 7.12, $L$ is a splitting projection. By the clifford property, $\left[T_{1} T_{2}, T_{0}\right]=0$, and analogously for $T_{3}$ and $T_{4}$. Note also that $V_{L}=-\exp \left(-i \pi J_{3}\right)$.

The linear span of the operators $\left\{L T_{0}, L T_{3}, L T_{4}\right\}$, acting on the range of $L$, is isomorphic to the set of operators $H(\mathbf{B})=\mathbf{B} \cdot \mathbf{J}$ for $J=1 / 2$. Thus follows by noting that both sets span the simple traceless 2 by 2 complex matrices. Alternatively, only could use the Clifford properties of $\left\{L T_{0}, L T_{3}, L T_{4}\right\}$ to identify them with the $\sigma$ matrices. The Chern numbers of the resulting complex line bundles over $S^{2}$ are +1 and -1 . The analogous statements obviously hold for $\left\{(1-L) T_{0},(1-L) T_{3}\right.$, $\left.(1-L) T_{4}\right\}$. The adiabatic time evolution is now easily computed as in Sect. 1.2. The analog of Berry's phase is again a unit quaternion. In the basis (2.26), we obtain;

Proposition 7.14. The holonomy $U(t) P(0)$ for a closed path $c(t)$ on the split sphere spanned by $\left\{T_{0}, T_{3}, T_{4}\right\}$ is equal to $\exp ( \pm i \Lambda)$. Here $\Lambda$ is the solid angle on the split sphere subtended by the path, and $i$ is the unit quaternion. The sign depends on the energy eigenvalue.

Proof. By Lemma 7.12, the adiabatic time evolution operator $U(t)$ commutes with $V_{L}=T_{1} T_{2}$

$$
V_{L}=\left(\begin{array}{ll}
i & 0 \\
0 & i
\end{array}\right) .
$$

For $U(t)$ to commute with $V_{L}$, the entries the matrix of $U(t)$ in the same basis must be linear combinations of 1 and $i$. The above observations relating the split sphere to $H(\mathbf{B})$ complete the proof.

By the $S p(2)$ symmetry, we can now find split spheres for which the holonomy is any unit quaternion. For example, the split sphere consisting of real Hamiltonians, spanned by $\left\{T_{0}, T_{2}, T_{3}\right\}$, has holonomy $\exp ( \pm j \Lambda)$. Note that $V= \pm \exp \left(-i \pi J_{2}\right)$ for this split sphere.

For $J>3 / 2$, the symmetry group is smaller, but it is still easy to find split spheres. $V_{L}=\exp (-i \pi \hat{\mathbf{n}} \cdot \mathbf{J})$, where $\hat{\mathbf{n}}$ is any unit vector, satisfies the necessary properties in Lemma 7.12. All such split spheres are related by the $S U(2)$ symmetry, so it suffices to consider the split sphere corresponding to $V_{L}=\exp \left(-i \pi J_{3}\right)$, which is spanned by $\left\{T_{0}, T_{3}, T_{4}\right\}$. We note that for $J>3 / 2$, this split sphere does not have a transitive symmetry group, but $-i J_{3}$ generates an axial symmetry. This 
symmetry is sufficient to apply the result of Sect. 1.2. We can thus obtain the first Chern number of each of complex line bundles over the split sphere. Note that $\exp \left(-i \pi J_{3}\right)$ is the identity transformation on the split sphere, whereas in Sect. 1.2 it was $\exp \left(-i 2 \pi J_{3}\right)$. Using Eq. (1.23), and denoting by $P_{T}$ and $P_{B}$ the appropriate spectral projections of $T_{0}$ and $-T_{0}$ respectively, we find for the first Chern class of the complex line bundle $L P$ over $S^{2}$

$$
C_{1}=\operatorname{Tr}\left(L P_{T} \frac{1}{2} J_{3}\right)-\operatorname{Tr}\left(L P_{B} \frac{1}{2} J_{3}\right) \text {. }
$$

$L$ projections onto the complex subspace spanned by the $J_{3}$ eigenstates $\{|J, 1 / 2\rangle,|J,-3 / 2\rangle,|J,+5 / 2\rangle \ldots\}$. We then obtain the first Chern numbers for these split spheres. For $J=3 / 2$, they are $+1,-1$, as determined earlier. For $J=5 / 2$, we obtain $\{+1,0,-1\}$, for $J=7 / 2$, the numbers are $\{2,-2,2,-2\}$, etc. One immediate use of these numbers is to compute the holonomy for the closed path $\cos (t) T_{0}+\sin (t) T_{3}$. The holonomy is +1 if the corresponding first Chern number is even, -1 if it is odd.

Another class of split spheres for $J>3 / 2$ is defined by

$$
V_{L}=i \operatorname{sign}(\hat{\mathbf{n}} \cdot \mathbf{J}) \equiv i \frac{\hat{\mathbf{n}} \cdot \mathbf{J}}{|\hat{\mathbf{n}} \cdot \mathbf{J}|} .
$$

Again, all such split spheres are related by the $S U(2)$ symmetry, and it suffices to consider $V_{L}=i \operatorname{sign}\left(J_{3}\right)$, which is spanned by $\left\{T_{0}, T_{1}, T_{2}\right\}$. These split spheres also have an axial symmetry, and the first Chern numbers can be calculated by the same method.

One can further compute the holonomy for closed paths on split spheres using the formulae of Sect. 1.2, Eq. (1.24) in particular. The complex one-dimensional projections are of course $L P_{\theta}$, where $P_{\theta}$ are the spectral projections for $T_{\theta}$, and one must use $\frac{1}{2} J_{3}$ instead of $J_{3}$ for the $V_{L}=\exp (-i \pi \hat{\mathbf{n}} \cdot \mathbf{J})$ class of split sphere. This is straightforward if the spectral projections $P_{\theta}$ are known. The spectral projections for $J=5 / 2$ are calculated in the appendix.

The time evolution for open paths on split spheres can also be calculated explicitly, by closing paths with segments whose time evolutions can be calculated. This is straightforward, and we do not discuss it here.

\section{Appendix: $J=5 / 2$ Spectral Projections}

Denote by $T_{\theta}=\cos (\theta) T_{0}+\sin (\theta) T_{3}$. In the basis (2.26), abbreviating $c=\cos (\theta)$ and $s=\sin (\theta)$,

$$
T_{\theta}=\frac{1}{3}\left(\begin{array}{ccc}
10 c & \sqrt{30} s & 0 \\
\sqrt{30} s & -8 c & 3 \sqrt{6} s \\
0 & 3 \sqrt{6} s & -2 c
\end{array}\right) .
$$

We already know from Proposition 3.5 that $T_{\theta}$ is traceless, and $\operatorname{Tr}\left(T_{\theta}^{2}\right)$ is independent of $\theta . T_{\theta}$ is then a quadrupole matrix, and we may use the results of Sect. 3.1. One checks that $\frac{3}{2} \operatorname{Tr}\left(T_{\theta}^{2}\right)=28$, so $B_{\theta}=T_{\theta} / \sqrt{28}$ is a unit quadrupole matrix. Using Proposition 3.1, we find that $T_{\theta}$ is related by $S O(3)$ conjugation to 
a matrix of the form

$$
\sqrt{28 \frac{2}{3}}\left(\begin{array}{lll}
\cos (\phi+2 \pi / 3) & & \\
& \cos (\phi-2 \pi / 3) & \\
& & \cos (\phi)
\end{array}\right)=\left(\begin{array}{lll}
E_{1} & & \\
& E_{2} & \\
& & E_{3}
\end{array}\right)
$$

for a unique $0 \leqq \phi \leqq \pi / 3$. Note further that $E_{1} \leqq E_{2} \leqq E_{3}$. Of course $T_{\theta}$ is nondegenerate, so $E_{1}<E_{2}<E_{3}$. Furthermore, from the proof of Proposition 3.1, we see that $\operatorname{Det}\left(B_{\theta}\right)=\frac{2}{27} \cos (3 \phi)$. It is straightforward to compute $\operatorname{Det}\left(T_{\theta}\right)=$ $\left(c^{3}-3 c s^{2}\right) 160 / 27=\cos (3 \theta) 160 / 27$. The eigenvalues of $T_{\theta}$ thus are

$$
\begin{gathered}
E_{1}=\frac{4 \sqrt{7}}{3} \cos \left(\frac{1}{3} \cos ^{-1}\left[\frac{10}{(7)^{3 / 2}} \cos (3 \theta)\right]+2 \pi / 3\right), \\
E_{2}=\frac{4 \sqrt{7}}{3} \cos \left(\frac{1}{3} \cos ^{-1}\left[\frac{10}{(7)^{3 / 2}} \cos (3 \theta)\right]-2 \pi / 3\right), \\
E_{3}=\frac{4 \sqrt{7}}{3} \cos \left(\frac{1}{3} \cos ^{-1}\left[\frac{10}{(7)^{3 / 2}} \cos (3 \theta)\right]\right) .
\end{gathered}
$$

We can now solve for the spectral projections. Real normalized eigenvectors of $B_{\theta}$ are

$$
\left|E_{n}\right\rangle=\frac{1}{C}\left(\beta_{n}|5 / 2\rangle+\gamma_{n}|-3 / 2\rangle+|1 / 2\rangle\right)
$$

and $\Theta\left|E_{n}\right\rangle$. Here

$$
\beta_{n}=\frac{\sqrt{30} s}{E_{n}-10 c}, \quad \gamma_{n}=\frac{3 \sqrt{6} s}{E_{n}+2 c}
$$

are functions of $\theta$, as is the normalization constant $C$, determined by

$$
C_{n}^{2}=\frac{30 s^{2}}{\left(E_{n}-10 c\right)^{2}}+\frac{45}{\left(E_{n}+2 c\right)^{2}}+1 \text {. }
$$

\section{References}

1. Berry, M.V.: Proc. Roy. Soc. London A 392, 45 (1984)

2. Simon, B.: Phys. Rev. Lett. 51, 2167 (1983)

3. Wilczek, F., Zee, A.: Phys. Rev. Lett. 52, 2111 (1984)

4. Mead, C. A.: Phys. Rev. Lett. 59, 161 (1987)

5. Avron, J., Sadun, L., Segert, J., Simon, B.: Phys. Rev. Lett. 61, 1329 (1988)

6. Avron, J. E., Seiler, R., Yaffe, L. G.: Commun. Math. Phys. 110, 110 (1987)

7. Kato, T.: J. Phys. Soc. J. Jpn. 5, 435 (1950)

8. Choquet-Bruhat, Y., DeWitt-Morette, C., Dillard-Bleick, M.: Analysis, Manifolds and Physics, rev ed. Amsterdam: North Holland 1982

9. Kobayashi, S., Nomizu, K.: Foundations of differential geometry, vol. I and II. New York: John Wiley 1963,1969

10. Atiyah, M.F.: Geometry of Yang-Mills fields, Lezioni Fermiane. Pisa: Accademia Nazionale dei Lincei \& Scuola Normale Superiore 1979 
11. Chern, S. S.: Complex manifolds without potential theory, sec. ed. Berlin, Heidelberg, New York: Springer 1979

12. Zee, A.: Phys. Rev. A 38, 1 (1988)

13. Tinkham, M.: Group theory and quantum mechanics. New York: McGraw-Hill 1964

14. Bjorken, J. D., Drell, S. D.: Relativistic quantum mechanics. New York: McGraw-Hill 1964; Itzykson, C., Zuber, J. B.: Quantum field theory. New York: McGraw-Hill 1980

15. Wigner, E. P.: Göttinger Nachr. 31, 546 (1932); Wigner, E. P.: Group Theory. New York: Academic Press 1959

16. Frobenius, Schur: Berl. Ber., p. 186 (1906)

17. Dyson, F.: J. Math. Phys. 3, 140 (1964)

18. Mehta, M. L.: Random matrices and the statistical theory of energy levels. New York: Academic Press 1967

19. Kramers, H.A.: Proc. Acad. Amsterdam 33, 959 (1930)

20. Adams, J.F.: Lectures on Lie Groups. Chicago: University of Chicago Press 1969

21. Bröcker, T., tom Dieck, T.: Representations of compact Lie groups. Berlin, Heidelberg, New York: Springer 1985

22. Sadun, L., Segert, J.: J. Phys. A 22, L 111 (1989)

23. von Neumann, J., Wigner, E.P.: Phys. Zeit. 30, 467 (1929)

24. Friedland, S., Robbin, J. W., Sylvester, J. H.: On the crossing rule. Commun. Pure Appl. Math. 37, 19 (1984)

25. Mermin, N. D.: Rev. Mod. Phys. 51, No. 3 (1979)

26. Avron, J., Seiler, R., Simon, B.: Phys. Rev. Lett. 51, 51 (1983)

27. Steenrod, N.: The topology of fibre bundles. Princeton: Princeton University Press 1951

28. Bott, R., Tu, L. W.: Differential forms in algebraic topology. Berlin, Heidelberg, New York: Springer 1982

29. Herzberg, G., Longuet-Higgins, H. C.: Discuss. Faraday Soc. 55, 77 (1963)

30. Milnor, J. W., Stasheff, J. D.: Characteristic classes. Princeton, NJ: Princeton University Press 1974

31. Thouless D., et. al.: Phys. Rev. Lett. 49, 405 (1982)

32. Kohmoto, K.: Ann. Phys. 160, 343 (1985)

33. Chang, L. N., Liang, Y.: Mod. Phys. L A 3, 1839 (1988)

34. Atiyah, M. F., Hitchin, N. J., Singer, I. M.: Proc. R. Soc. Lond. A 362, 425 (1978)

35. Karoubi, M., Leruste, C.: Algebraic topology via differential geometry. Cambridge: Cambridge University Press 1987

36. Wu. Y. S., Zee, A.: Phys. Lett. B 207, 39 (1988)

37. Chern, S.S., Simons, J.: Ann. Math. 99, 48 (1974)

38. Moody, J., Shapere, A., Wilczek, F.: Phys. Rev. Lett. 56, 893 (1986)

39. Tycko R.: Phys. Rev. Lett. 58, 2281 (1987)

40. Segert, J.: Math. Phys. 28, 2102 (1987)

Communicated by A. Jaffe

Received December 1, 1988; in revised form February 22, 1989 
